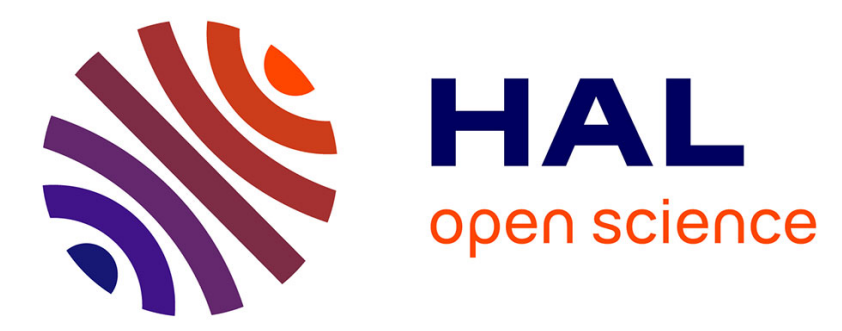

\title{
Probabilistic approach for model and data uncertainties and its experimental identification in structural dynamics: Case of composite sandwich panels
}

\author{
C. Chen, Denis Duhamel, Christian Soize
}

\section{- To cite this version:}

C. Chen, Denis Duhamel, Christian Soize. Probabilistic approach for model and data uncertainties and its experimental identification in structural dynamics: Case of composite sandwich panels. Journal of Sound and Vibration, 2006, 294 (1-2), pp.64-81. 10.1016/j.jsv.2005.10.013 . hal-00686153

\section{HAL Id: hal-00686153 https://hal.science/hal-00686153}

Submitted on 7 Apr 2012

HAL is a multi-disciplinary open access archive for the deposit and dissemination of scientific research documents, whether they are published or not. The documents may come from teaching and research institutions in France or abroad, or from public or private research centers.
L'archive ouverte pluridisciplinaire HAL, est destinée au dépôt et à la diffusion de documents scientifiques de niveau recherche, publiés ou non, émanant des établissements d'enseignement et de recherche français ou étrangers, des laboratoires publics ou privés. 


\section{JSV REFERENCE: P/284/04}

TITLE: Probabilistic approach for model and data uncertainties and its experimental identification in structural dynamics: case of composite sandwich panels.

AUTHORS: C. Chen ${ }^{(1)}$, D. Duhamel ${ }^{(2)}$, C. Soize $(1)$

(1) University of Marne-la-Vallée, Laboratoire de Mécanique, Institut Navier, 5 boulevard Descartes, 77454 Marne-la-Vallée, France

(2) Ecole Nationale des Ponts et Chaussées, Laboratoire Analyse Matériaux et Identification, Institut Navier, 6-8 avenue Blaise Pascal, 77455 Marne-la-Vallée, France

SUBMITTED TO THE JOURNAL OF SOUND AND VIBRATION: November 2004 FIRST REVISED VERSION: June 2005

SECOND REVISED VERSION: October 2005

CORRESPONDING AUTHOR:

C. Soize

Laboratoire de Mécanique

Université de Marne-la-Vallée

5 Bd Descartes

77454 Marne-la-Vallée Cedex 2

France

E-MAIL: soize@univ-mlv.f

FAX: 33160957799

TOTAL NUMBER OF PAGES: 29

TOTAL NUMBER OF FIGURES: 13

C. Chen, D. Duhamel, C. Soize, second revision October 2005, first revision June 2005, submitted in October 2004 to JSV 


\begin{abstract}
This paper deals with the experimental identification and the validation of a non-parametric probabilistic approach allowing model uncertainties and data uncertainties to be taken into account in the numerical model developed to predict low- and medium-frequency dynamics of structures. The analysis is performed for a composite sandwich panel representing a complex dynamical system which is sufficiently simple to be completely described and which exhibits, not only data uncertainties, but above all model uncertainties. The dynamical identification is experimentally performed for 8 panels. The experimental frequency response functions are used to identify the non-parametric probabilistic approach of model uncertainties. The prediction of the low- and medium-frequency dynamical responses obtained with the stochastic system is compared with the experimental measurements.
\end{abstract}

Keywords: uncertainties, experimental identification, structural dynamics, composite, sandwich panel

\title{
1. Introduction
}

The last two decades have received a particular attention in developments of parametric probabilistic approach for modelling data uncertainties (material properties, geometry, boundary conditions) in structural dynamics, for many simple and complex dynamical systems, including the case of composite structures (see for instance, Refs. [1-9] for analysis, optimal design, stability analysis, free vibration and reliability analysis of composite structures).

This paper has two main objectives. The first one is to present the validation of an experimental identification method of a general non-parametric probabilistic approach recently introduced (see Refs.[10,11,12]), allowing model and data uncertainties to be taken into account in structural dynamics. The structure which has been chosen for performing this probabilistic analysis is a composite sandwich panel because it constitutes a complex dynamical system which is sufficiently simple to be completely described and which exhibits not only data uncertainties but above all model uncertainties. The second objective is to analyse the role played by model uncertainties in the dynamical responses of such mechanical system. It is known that the dynamical responses of light composite sandwich panels in the medium-frequency range are sensitive to the process used for their manufacturing. In addition, such sandwich panels constitute complex dynamical systems (dynamical behavior of the materials consituting the different layers; interface conditions between two adjacent layers; boundary conditions, etc) and consequently, model uncertainties are induced by the mathematical-mechanical modelling 
process in which simplifications are introduced. Finally, the parameters of the mathematicalmechanical modelling are not known with a great precision which means that data parameters are also uncertain. It should be noted that this paper addresses (1) neither uncertain loads, (2) neither data uncertainties modelled by perturbation techniques or by the usual parametric probabilistic approach (3) nor active control and related topics such as synthesis of active controllers. This paper mainly addresses a new experimental validation of a general probabilistic approach which allows model uncertainties and data uncertainties to be taken into account in the numerical predictive models for the low- and medium-frequency dynamics. Eight sandwich panels have been manufactured using an identical process and their frequency response functions have been experimentally identified.

The designed composite sandwich panel is constituted of two thin carbon-resin skins and one high stiffness closed-cell foam core. Each skin is constituted of 2 unidirectional plies [60/60]. As written above, it is known that such sandwich panels, manufactured with an identical process, generally present a significant dispersion for their Frequency Response Functions (FRF) in the low-requency (LF) range and above all in the medium-frequency (MF) range. Concerning the sandwich panel, the objectives are (1) to perform an experimental analysis of the frequency-response-functions dispersion due to the process used for manufacturing the sandwich panels, (2) to develop a predictive mean mechanical model based on the use of the laminated composite thin plate theory in dynamics and to compare the numerical simulations with the experiments, and (3) to use a non-parametric probabilistic approach allowing data and model uncertainties to be modelled in order to improve the predictability of the mean model in the LF and MF dynamics.

The non-parametric probabilistic approach used in this paper is based on the concepts and the methodology introduced in Refs. [10,11,12]. In such a probabilistic model, the probability distribution of each full random generalized matrix of the dynamical system (generalized mass, damping and stiffness matrices) depends on a dispersion parameter (the coefficient of variation of the full random matrix constructed with the Frobenius norm) allowing the level of the random fluctuations of each random matrix to be controlled. An experimental estimation of each dispersion parameter for the random generalized mass, damping and stiffness matrices is proposed. The confidence regions of the random frequency response functions are predicted by using the random dynamical system constructed with the non-parametric probabilistic approach of model and data uncertainties and are compared with the experimental frequency response functions measured for the 8 sandwich panels. 


\section{Notation}

In this paper, the following notations are used:

(1) A lower case letter is a real or complex deterministic variable (e.g. $f$ ).

(2) A boldface lower case letter is a real or complex deterministic vector (e.g. $\mathbf{f}=\left(f_{1}, \ldots, f_{n}\right)$.

(3) An upper case letter is a real or complex random variable (e.g. F).

(4) A boldface upper case letter is a real or complex random vector (e.g. $\mathbf{F}=\left(F_{1}, \ldots, F_{n}\right)$ ).

(5) An upper case letter between brackets is a real or complex deterministic matrix (e.g. $[A]$ ).

(6) A boldface upper case letter between brackets is a real or complex random matrix (e.g. $[\mathbf{A}])$.

(7) Any deterministic quantities above (e.g. $f, \mathbf{f},[A])$ with an underline (e.g. $\underline{f}, \underline{\mathbf{f}},[\underline{A}])$ means that these deterministic quantities are related to the mean model (or to the nominal model).

In addition, the following algebraic notations are used.

Euclidean space. The Euclidean space $\mathbb{R}^{m}$ is equipped with the usual inner product such that, for all $\mathbf{u}=\left(u_{1}, \ldots, u_{m}\right)$ and $\mathbf{v}=\left(v_{1}, \ldots, v_{m}\right)$ in $\mathbb{R}^{m},\langle\mathbf{u}, \mathbf{v}\rangle=u_{1} v_{1}+\ldots+u_{m} v_{m}$ and the associated norm $\|\mathbf{u}\|=<\mathbf{u}, \mathbf{u}>^{1 / 2}$. The bilinear form $(\mathbf{u}, \mathbf{v}) \mapsto<\mathbf{u}, \mathbf{v}>$ is extended to complex vectors $\mathbf{u}$ and $\mathbf{v}$ belonging to the Hermitian space $\mathbb{C}^{m}$.

Hermitian space. For all $\mathbf{u}=\left(u_{1}, \ldots, u_{m}\right)$ in the Hermitian space $\mathbb{C}^{m}$, its hermitian norm is such that $\|\mathbf{u}\|=\left\{\left|u_{1}\right|^{2}+\ldots+\left|u_{m}\right|^{2}\right\}^{1 / 2}$.

Matrix sets. Let $M_{n, m}(\mathbb{R})$ be the set of all the $(n \times m)$ real matrices, $M_{n}(\mathbb{R})=\mathbb{M}_{n, n}(\mathbb{R})$ be the set of all the square $(n \times n)$ real matrices, $\mathbb{M}_{n}^{S}(\mathbb{R})$ be the set of all the $(n \times n)$ real symmetric matrices and $M_{n}^{+}(\mathbb{R})$ be the set of all the $(n \times n)$ real symmetric positive-definite matrices. We then have $\mathbb{M}_{n}^{+}(\mathbb{R}) \subset \mathbb{M}_{n}^{S}(\mathbb{R}) \subset \mathbb{M}_{n}(\mathbb{R})$.

Norms and usual operators. We denote:

(1) the determinant of matrix $[A] \in \mathbb{M}_{n}(\mathbb{R})$ as $\operatorname{det}[A]$ and its trace as $\operatorname{tr}[A]=\sum_{j=1}^{n}[A]_{j j}$,

(2) the transpose of $[A] \in \mathbb{M}_{n, m}(\mathbb{R})$ as $[A]^{T} \in \mathbb{M}_{m, n}(\mathbb{R})$,

(3) the Frobenius norm (or Hilbert-Schmidt norm) $\|A\|_{F}$ of $[A]$ as $\|A\|_{F}=\left\{\operatorname{tr}\left\{[A]^{T}[A]\right\}\right\}^{1 / 2}$.

(4) the mathematical expectation of any random quantity such as $[\mathbf{A}]$ is denoted by $E\{[\mathbf{A}]\}$.

\section{Description of the designed sandwich panel}

The designed sandwich panel is constituted of five layers made of four thin carbon-resin unidirectional plies and one high stiffness closed-cell foam core. This panel is defined with respect to a Cartesian coordinate system $O x y z$ and is $0.40 \mathrm{~m}$ length ( $O x$ axis), $0.30 \mathrm{~m}$ width $(O y$ axis) and $0.01068 \mathrm{~m}$ total thickness ( $O z$ axis). The middle plane of the sandwich panel is 
$O x y$ and the origine $O$ is located in the corner. Each carbon layer is made of a thin carbon-resin ply with a thickness of $0.00017 \mathrm{~m}$, a mass density $\rho=1600 \mathrm{Kg} / \mathrm{m}^{3}$. Let $O X Y Z$ be the local Cartesian coordinate system attached to a carbon-resin ply for which $O X Y$ coincides with the plan of the ply and for which its fibers are oriented in $O X$ direction. Then, the elasticity constants expressed in the local coordinate system $O X Y Z$ are: $E_{X}=101 \mathrm{GPa}, E_{Y}=6.2$ $\mathrm{GPa}, \nu_{X Y}=0.32, G_{X Y}=G_{X Z}=G_{Y Z}=2.4 \mathrm{GPa}$. The first two layers are two carbonresin unidirectional plies in a [-60/60] layup. The third layer is a closed-cell foam core with a thickness of $0.01 \mathrm{~m}$, a mass density of $80 \mathrm{Kg} / \mathrm{m}^{3}$ and elasticity constants: $E_{x}=E_{y}=60 \mathrm{MPa}$, $\nu_{x y}=0, G_{x y}=G_{x z}=G_{y z}=30 \mathrm{MPa}$. The fourth and fifth layers are two carbon-resin unidirectional plies in a [60/-60] layup.

\section{Manufacturing the sandwich panels}

Eight sandwich panels have been manufactured from the designed sandwich panel using an identical process and the same materials. All the sandwich panels have been baked in the same batch for suppressing the influences of the different baking conditions concerning time and temperature. The different steps for the manufacturing of the sandwich panels are the following. Step 1: cut out the carbon-resine tissue and cut out the foam plate with the dimension of the designed panel. Step 2: for each plate, paste the carbon-resine tissues with the foam plate. Step 3: bake the eight sandwich panels pasted in the previous step in the vacuum oven for solidify the oxygen resin existing in the sandwich. Figure 1 shows step 2 of the manufacturing process for a sandwich panel.

\section{Dynamical identification of the eight sandwich panels}

\subsection{Description of dynamical testing}

The panel is vertical and suspended by two thin soft rubber bands attached to the two upper corners of the panel. The eigenfrequency of the vertical body motion is about $2 \mathrm{~Hz}$ which has to be compared to the lowest elastic eigenfrequency which is $191 \mathrm{~Hz}$. Consequently, the measurements of the frequency response functions in the frequency band of analysis are then performed for a configuration corresponding to free-free conditions. The frequency band of analysis considered is the band $B=[10,4500] \mathrm{Hz}$ corresponding to the model validity of the mean finite element model. The input $z$-force is a point load applied to the point $N 0$ of coordinates $(0.187,0.103,0) \mathrm{m}$ and is delivered by an electrodynamic shaker which is horizontally fixed. The input force is measured with a force transducer which is located between the panel and the shaker. The experimental configuration used guarantees a correct 
excitation in bending mode with a driven force which can be modelled by a point force. Point $N 0$ has been chosen such that all the symmetric and anti-symmetric elastic modes of the panel is excited in the frequency band of analysis.

The output $z$-accelerations are measured at 25 points by accelerometers. For the sake of briefness, the presentation is limited to the 3 following points: $N 1$ with coordinates $(0.337,0.103,0) \mathrm{m}, N 2$ with coordinates $(0.112,0.159,0) \mathrm{m}$ and $N 3$ with coordinates $(0.337$, $0.216,0) \mathrm{m}$. The cross frequency response functions are identified on frequency band $B$ by using the usual spectral analysis method and signal processing $[13,14]$.

\subsection{Experimental cross frequency response functions}

Figures 2, 3 and 4 display the graphs of the modulus of the experimental cross frequency response functions in $\log$ scale for an input at point $N 0$ (driven point) and a transversal acceleration output at points $N 1, N 2$ and $N 3$, respectively. There are 8 graphs on each figure corresponding to the 8 sandwich panels. The analysis of the 25 experimental cross frequency response functions on frequency band $B=[10,4500] \mathrm{Hz}$ (in which there are 60 elastic modes) shows a small dispersion in the frequency band $[10,1550] \mathrm{Hz}$ (in which there are 11 elastic modes) and a significant dispersion, increasing with the frequencies, in the frequency band $[1550,4500] \mathrm{Hz}$ (in which there are about 59 elastic modes). This can clearly be seen in figures 2, 3 and 4 relative to points $N 1, N 2$ and $N 3$ respectively.

\subsection{Experimental modal analysis}

For each sandwich panel, an experimental modal analysis [15] has been performed using a commercialized software [16] in the frequency band $[10,1550] \mathrm{Hz}$ and the identified experimental cross frequency response functions (see Section 4.2). For each sandwich panel $r=1, \ldots, 8$, eleven elastic modes have been identified in this frequency band. For sandwich panel $r$, the following usual modal parameters of each experimental elastic mode $\alpha$ has been identified: (1) the eigenfrequency $\omega_{\alpha}^{\exp }\left(\theta_{r}\right),(2)$ the damping rate $\xi_{\alpha}^{\exp }\left(\theta_{r}\right),(3)$ the elastic mode shape $\psi_{\alpha}^{\exp }\left(\theta_{r}\right)$ and the corresponding generalized mass $\mu_{\alpha}^{\exp }\left(\theta_{r}\right)$.

The experimental modal model identification used to estimate the eigenfrequencies, the damping rates, the elastic mode shapes and the generalized masses (from data constituted of the experimental cross frequency response functions) are the following [16]: The identification procedure is to seek an approximation of the measured cross frequency response functions in the pole / residue usual form. An iterative refinement of the poles of the current model is performed. The three main steps of the procedure are: (1) finding initial pole estimates, adding missed poles, removing computational poles, (2) estimating residues and residual terms 
for a given set of poles, (3) optimizing poles and residues of the current model using a narrow frequency band update. In particular, from the poles, it is deduced the experimental eigenfrequencies and the experimental damping rates. This procedure allows the first eleven experimental eigenmodes to be identified without significant errors while the errors increase with the upper experimental eigenmodes (twelve, thirteenth, etc...). Consequently, only the first eleven identified eigenmodes have been kept.

Concerning the updating of the conservative part of the mean model with the first experimental eigenfrequencies (see Section 5.3), an average value of each experimental eigenfrequency is constructed over the set of the eight experimental panels. For each experimental eigenfrequency $\alpha$, the usual estimation $\underline{\omega}_{\alpha}^{\exp }$ defined by

$$
\underline{\omega}_{\alpha}^{\exp }=(1 / 8) \sum_{r=1}^{8} \omega_{\alpha}^{\exp }\left(\theta_{r}\right)
$$

is then introduced and represents the average experimental eigenfrequency. In addition, the updating of the conservative part of the mean model will be performed using only the first four "well isolated" eigenmodes. Introducing $\underline{f}_{\alpha}^{\exp }=\underline{\omega}_{\alpha}^{\exp } /(2 \pi)$, the results for the first four eigenfrequencies are

$$
\underline{f}_{1}^{\exp }=191.0 \mathrm{~Hz} \quad, \quad \underline{f}_{2}^{\exp }=329.5 \mathrm{~Hz} \quad, \quad \underline{f}_{3}^{\exp }=532.0 \mathrm{~Hz} \quad, \quad \underline{f}_{4}^{\exp }=635.1 \mathrm{~Hz} \quad .
$$

Concerning the dissipative part of the mean model no updating is performed to "obtain a good fit" (which would be really difficult to construct because the prediction performed is a confidence region of the stochastic frequency response functions corresponding to a given probability level and in addition, would be without any interest since the objective of the paper is to take into account model and data uncertainties and not only data uncertainties). A global average experimental damping rate is then constructed as explained below and then the average value is directly used in the mean model (see Section 5.2). For $\alpha=1, \ldots, 11$, let

$$
\underline{\xi}_{\alpha}^{\exp }=(1 / 8) \sum_{r=1}^{8} \xi_{\alpha}^{\exp }\left(\theta_{r}\right)
$$

be the average experimental damping rate $\alpha$ over the set of the eight experimental panels. Let

$$
\underline{\xi}^{\exp }=(1 / 11) \sum_{\alpha=1}^{11} \underline{\xi}_{\alpha}^{\exp }
$$

be the global average experimental damping rate for the first eleven experimental eigenfrequencies. This procedure yields the value

$$
\underline{\xi}^{\exp }=0.01
$$

which will be directly used in the mean model. 


\section{Mean mechanical model of the dynamical system and experimental comparisons}

The mean model refers to the model deduced from the mechanical-mathematical model of the designed sandwich panel for which data (geometry, elasticity constants, mass densities, etc) correspond to the designed sandwich panel data values and are usually called the mean data or the nominal data.

\subsection{Mean finite element model}

The designed panel is considered as a laminated composite thin plate for which each layer is made of an orthotropic elastic material $[17,18,19]$. The elasticity constants of each layer are given in Section 2. Since we are interested in the $z$-dispacement of the middle plane of the sandwich panel in the bending mode and since the panel is a free structure, there are 3 rigid body modes. We are interested in the construction of the responses in the frequency domain over the frequency band of analysis $B$. The designed panel is modelled by using a regular finite element meshes constituted of $64 \times 64$ four-nodes finite elements for laminated plate bending. The damping of the structure is introduced by an arbitrary usual model controlled by the modal damping rates (see section 5.2)

In frequency band $B$, the mean finite element model of linear vibrations of the free designed panel around a position of static equilibrium taken as reference configuration without prestresses is written as

$$
\left(-\omega^{2}[\underline{\mathbb{M}}]+i \omega[\underline{\mathbb{D}}]+[\underline{\mathbb{K}}]\right) \underline{\mathbf{y}}(\omega)=\underline{\mathbf{f}}(\omega) \quad, \quad \omega \in B \quad,
$$

in which $\underline{\mathbf{y}}(\omega)=\left(\underline{y}_{1}(\omega), \ldots, \underline{y}_{m}(\omega)\right)$ is the $\mathbb{C}^{m}$-vector of the $m$ DOFs (displacements and rotations) and $\underline{\mathbf{f}}(\omega)=\left(\underline{f}_{1}(\omega), \ldots, \underline{f}_{m}(\omega)\right)$ is the $\mathbb{C}^{m}$-vector of the $m$ inputs (forces and moments). The mean mass matrix $[\underline{M}]$ is a positive-definite symmetric $(m \times m)$ real matrix. The mean damping and stiffness matrices $[\underline{\mathbb{D}}]$ and $[\underline{\mathbb{K}}]$ are positive-semidefinite symmetric $(m \times m)$ real matrices (free structure). Matrices $[\underline{\mathbb{D}}]$ and $[\underline{\mathbb{K}}]$ have the same null space having a dimension $m_{\text {rig }}=3$ and spanned by the rigid body modes $\left\{\underline{\varphi}_{-2}, \underline{\varphi}_{-1}, \underline{\varphi}_{0}\right\}$. It is assumed that the given deterministic load vector $\underline{\mathbf{f}}(\omega)$ is in equilibrium, i.e. is such that $\left\langle\underline{\mathbf{f}}(\omega), \underline{\varphi}_{1-\beta}\right\rangle=0$ for all $\beta$ in $\{1,2,3\}$. For all $\omega$ in $B$, Eq. (1) has a unique solution $\underline{\mathbf{y}}(\omega)=[\underline{\mathbb{I}}(\omega)] \underline{\mathbf{f}}(\omega)$ in which $[\underline{\mathbb{I}}(\omega)]$ is the matrix-valued FRF (frequency response function) defined by $[\underline{\mathbb{T}}(\omega)]=[\underline{\mathbb{A}}(\omega)]^{-1}$ where $[\underline{A}(\omega)]$ is the dynamic stiffness matrix such that

$$
[\underline{\mathbb{A}}(\omega)]=-\omega^{2}[\underline{\mathbb{M}}]+i \omega[\underline{\mathbb{D}}]+[\underline{\mathbb{K}}] .
$$




\subsection{Mean reduced matrix model}

The mean reduced matrix model adapted to frequency band $B$ is constructed by using the usual modal analysis with the elastic modes of the associated conservative system. The generalized eigenvalue problem associated with the mean mass and stiffness matrices of the mean finite element model is written as $[\underline{\mathbb{K}}] \underline{\varphi}=\underline{\lambda}[\underline{M}] \underline{\varphi}$. Since $[\underline{\mathbb{K}}]$ is a positive-semidefinite matrix, we have $\underline{\lambda}_{-2}=\underline{\lambda}_{-1}=\underline{\lambda}_{0}=0<\underline{\lambda}_{1} \leq \underline{\lambda}_{2} \leq \ldots \leq \underline{\lambda}_{m}$ and the associated elastic modes $\left\{\underline{\varphi}_{1}, \underline{\varphi}_{2}, \ldots\right\}$ corresponding to the strictly positive eigenvalues $\underline{\lambda}_{1}, \underline{\lambda}_{2}, \ldots$, are such that $<[\underline{M}] \underline{\varphi}_{\beta}, \underline{\varphi}_{\beta^{\prime}}>=\underline{\mu}_{\beta} \delta_{\beta \beta^{\prime}}$ and $<[\underline{\mathbb{K}}] \underline{\varphi}_{\beta}, \underline{\varphi}_{\beta^{\prime}}>=\underline{\mu}_{\beta} \underline{\omega}_{\beta}^{2} \delta_{\beta \beta^{\prime}}$ in which $\underline{\omega}_{\beta}=\sqrt{\underline{\lambda}_{\beta}}$ is the eigenfrequency of elastic mode $\underline{\varphi}_{\beta}$ whose normalization is defined by the generalized mass $\underline{\mu}_{\beta}$. The mean reduced matrix model of the dynamic system whose mean finite element model is defined by Eq. (1) is obtained by constructing the projection of the mean finite element model on the subspace $V_{n}$ of $\mathbb{R}^{m}$ spanned by $\left\{\underline{\varphi}_{1}, \ldots, \underline{\varphi}_{n}\right\}$ with $n \ll m$. Let $\left[\underline{\Phi}_{n}\right]$ be the $(m \times n)$ real matrix whose columns are vectors $\left\{\underline{\varphi}_{1}, \ldots, \underline{\varphi}_{n}\right\}$. The generalized mass, damping and stiffness matrices $\left[\underline{M}_{n}\right],\left[\underline{D}_{n}\right]$ and $\left[\underline{K}_{n}\right]$ are positive-definite symmetric $(n \times n)$ real matrices such that $\left[\underline{M}_{n}\right]_{\beta \beta^{\prime}}=\underline{\mu}_{\beta} \delta_{\beta \beta^{\prime}},\left[\underline{D}_{n}\right]_{\beta \beta^{\prime}}=<[\underline{\mathbb{D}}] \underline{\varphi}_{\beta^{\prime}}, \underline{\varphi}_{\beta}>$ and $\left[\underline{K}_{n}\right]_{\beta \beta^{\prime}}=\underline{\mu}_{\beta} \underline{\omega}_{\beta}^{2} \delta_{\beta \beta^{\prime}}$. In general, $\left[\underline{D}_{n}\right]$ is a full matrix. Nevertheless, as explained in section 5.1, the damping model is introduced in writing that $\left[\underline{D}_{n}\right]_{\beta \beta^{\prime}}=2 \underline{\xi}_{\beta} \underline{\mu}_{\beta} \underline{\omega}_{\beta} \delta_{\beta \beta^{\prime}}$ in which $\underline{\xi}_{1}, \ldots, \underline{\xi}_{n}$ are the mean modal damping rates. The mean damping model is then chosen (see Section 4.3) such that

$$
\underline{\xi}_{1}=\ldots=\underline{\xi}_{n}=\underline{\xi}^{\exp }=0.01
$$

For frequency band $B$, the mean reduced matrix model of the dynamic system is written as the approximation $\underline{\mathbf{y}}^{n}(\omega)$ of $\underline{\mathbf{y}}(\omega)$ such that

$$
\underline{\mathbf{y}}^{n}(\omega)=\left[\underline{\Phi}_{n}\right] \underline{\mathbf{q}}^{n}(\omega) \quad, \quad \omega \in B \quad,
$$

in which the $\mathbb{C}^{n}$-vector $\underline{\mathbf{q}}^{n}(\omega)$ of the generalized coordinates is the unique solution of the mean reduced matrix equation,

$$
\left(-\omega^{2}\left[\underline{M}_{n}\right]+i \omega\left[\underline{D}_{n}\right]+\left[\underline{K}_{n}\right]\right) \underline{\mathbf{q}}^{n}(\omega)=\underline{\mathcal{F}}^{n}(\omega) \quad, \quad \omega \in B
$$

with $\underline{\mathcal{F}}^{n}(\omega)=\left[\underline{\Phi}_{n}\right]^{T} \underline{\mathbf{f}}(\omega) \in \mathbb{C}^{n}$ and where the mean generalized mass, damping and stiffness matrices are the positive-definite symmetric $(n \times n)$ real diagonal matrices defined above. 


\subsection{Updating the conservative part of the mean model with the first experimental eigenfrequencies}

Firstly, the mean value of the mass density $\rho$ of each carbon-resine ply has been identified by using (1) a measurement of the dimensions and of the total weight of the panel and (2) the mass density of the foam given by the manufacturer. This identification yields $\rho^{\text {upd }}=1904 \mathrm{Kg} / \mathrm{m}^{3}$. Secondly, the Young moduli $E_{X}$ and $E_{Y}$ of each carbon-resin ply has been updated with respect to the first eigenfrequencies. The main hypothesis used is to obtain an updated mean model which has a correct global stiffness. Consequently, since only the two parameters $E_{X}$ and $E_{Y}$ are used for this updating, the number of elastic mode has been limited to the first four eigenfrequencies. The calculation of the eigenfrequencies of the designed panel with data defined in Section 2 has been performed with the mean finite element model (see Section 5.1) whose finite element mesh is made of $128 \times 64$ four-nodes finite elements. For this designed panel, the first four computed eigenfrequencies are

$$
\underline{f}_{1}=176.4 \mathrm{~Hz} \quad, \quad \underline{f}_{2}=344.8 \mathrm{~Hz} \quad, \quad \underline{f}_{3}=499.7 \mathrm{~Hz} \quad, \quad \underline{f}_{4}=651.2 \mathrm{~Hz} \quad .
$$

The updating of the conservative part of the mean model is then performed in minimizing the following cost function,

$$
J\left(E_{X}, E_{Y}\right)=\sum_{\beta=1}^{4}\left|\underline{f}_{\beta}-\underline{f}_{\beta}^{\exp }\right|,
$$

with respect to $E_{X}$ and $E_{Y}$, where

$$
\underline{f}_{1}^{\exp }=191.0 \mathrm{~Hz} \quad, \quad \underline{f}_{2}^{\exp }=329.5 \mathrm{~Hz} \quad, \quad \underline{f}_{3}^{\exp }=532.0 \mathrm{~Hz} \quad, \quad \underline{f}_{4}^{\exp }=635.1 \mathrm{~Hz} \quad,
$$

are the average experimental eigenfrequencies defined in Section 4.3, and where all the other mechanical parameters take the values defined in Section 2 except $\rho=\rho^{\text {upd }}$. The updated values for $E_{X}$ and $E_{Y}$ are

$$
E_{X}^{\text {upd }}=103 \mathrm{GPa} \quad, \quad E_{Y}^{\text {upd }}=6.0 \mathrm{GPa} \quad,
$$

and yields for the first four updated eigenfrequencies,

$$
\underline{f}_{1}^{\text {upd }}=191.7 \mathrm{~Hz} \quad, \quad \underline{f}_{2}^{\text {upd }}=332.8 \mathrm{~Hz} \quad, \quad \underline{f}_{3}^{\text {upd }}=529.5 \mathrm{~Hz} \quad, \quad \underline{f}_{4}^{\text {upd }}=630.8 \mathrm{~Hz} \quad .
$$

Below, the updated mechanical parameters are used instead of the values defined for the designed sanswich panel. The designed sandwich panel with the updated mechanical constants will be named the updated designed sandwich panel associated with the updated mean finite element model and the updated mean reduced matrix model. 


\subsection{Convergence with respect to the mesh size for the updated designed panel}

A convergence analysis of the cross frequency response functions of the updated designed sandwich panel has been performed with respect to the size mesh of the finite element mesh. Figure 5 displays the graphs of the cross FRF between point $N 0$ and point $N 1$ for the three finite element meshes: $32 \times 32$ four-nodes finite elements, $64 \times 64$ four-nodes finite elements and $128 \times 64$ four-nodes finite elements. All the results obtained, and in particular Figure 5, show that convergence with respect to the finite element mesh size is reasonable for $64 \times 64$ four-nodes finite elements.

\subsection{Convergence of the updated mean reduced matrix model with respect to the number of elastic modes}

The convergence with respect to the dimension of the updated mean reduced matrix model is analyzed in studing the graph of the $L^{2}$-norm in space (over all the middle plane of the sandwich panel) and in frequency (over all the frequency band of analysis $B$ ) of the $z$ acceleration response for a unit input applied to point $N 0$. Figure 6 displays the graph of this norm versus the dimension of the updated mean reduced matrix model, that is to say, versus the number of elastic modes. The convergence is reached for $n=120$.

\subsection{FRF calculation with the updated mean reduced matrix model and experimental comparisons}

The cross frequency response functions are calculated by using Eqs. (3) and (4) (updated mean reduced matrix model) with $n=200$. Figures 7,8 and 9 display the graphs of the modulus of the experimental and numerical cross frequency response functions in log scale for an input at point $N 0$ (driven point) and a $z$-acceleration output at points $N 1, N 2$ and $N 3$, respectively. There are 9 graphs on each figure: 8 graphs correspond to the experimental cross frequency response functions associated with the 8 sandwich panels and 1 graph corresponds to the numerical cross frequency response function computed with the updated mean reduced matrix model.

The comparisons of the experimental cross frequency response functions with those constructed with the updated mean finite element model are reasonably good in the frequency band $[0,1500]$ $\mathrm{Hz}$ and are relatively bad in $[1500,4500] \mathrm{Hz}$. In the frequency band $[1500,4500] \mathrm{Hz}$, the lack of predictability is increasing with the frequency and is mainly due to data uncertainties (mechanical parameters) and to model uncertainties (modelling the sandwich panel by using the laminated composite thin plate theory). 


\section{Nonparametric Model of Random Uncertainties}

The non-parametric model of random uncertainties has initially been introduced in Ref. [10]. The construction of the non-parametric model of random uncertainties in the frequency band $B$ consists in modelling the generalized mass, damping and stiffness matrices of the mean reduced matrix model defined by Eqs. (3) and (4) by full random matrices $\left[\mathbf{M}_{n}\right],\left[\mathbf{D}_{n}\right]$ and $\left[\mathbf{K}_{n}\right]$ with values in $\mathbb{M}_{n}^{+}(\mathbb{R})$ such that $E\left\{\left[\mathbf{M}_{n}\right]\right\}=\left[\underline{M}_{n}\right], E\left\{\left[\mathbf{D}_{n}\right]\right\}=\left[\underline{D}_{n}\right]$ and $E\left\{\left[\mathbf{K}_{n}\right]\right\}=\left[\underline{K}_{n}\right]$. Consequently, the non-parametric model of random uncertainties in frequency band $B$ is written as

$$
\mathbf{Y}^{n}(\omega)=\left[\underline{\Phi}_{n}\right] \mathbf{Q}^{n}(\omega)
$$

in which, for all $\omega$ fixed in $B$, the $\mathbb{C}^{n}$-valued random variable $\mathbf{Q}^{n}(\omega)$ of the random generalized coordinates is the unique solution of the random reduced matrix equation,

$$
\left(-\omega^{2}\left[\mathbf{M}_{n}\right]+i \omega\left[\mathbf{D}_{n}\right]+\left[\mathbf{K}_{n}\right]\right) \mathbf{Q}^{n}(\omega)=\underline{\mathcal{F}}^{n}(\omega) \quad, \quad \omega \in B
$$

From Refs. $[10,11,12,20]$, these random matrices are written as

$$
\begin{aligned}
{\left[\mathbf{M}_{n}\right] } & =\left[\underline{L}_{M_{n}}\right]^{T}\left[\mathbf{G}_{M_{n}}\right]\left[\underline{L}_{M_{n}}\right], \\
{\left[\mathbf{D}_{n}\right] } & =\left[\underline{L}_{D_{n}}\right]^{T}\left[\mathbf{G}_{D_{n}}\right]\left[\underline{L}_{D_{n}}\right], \\
{\left[\mathbf{K}_{n}\right] } & =\left[\underline{L}_{K_{n}}\right]^{T}\left[\mathbf{G}_{K_{n}}\right]\left[\underline{L}_{K_{n}}\right],
\end{aligned}
$$

in which the positive-definite $(n \times n)$ real diagonal matrices $\left[\underline{L}_{M_{n}}\right],\left[\underline{L}_{D_{n}}\right]$ and $\left[\underline{L}_{K_{n}}\right]$ are such that $\left[\underline{M}_{n}\right]=\left[\underline{L}_{M_{n}}\right]^{2},\left[\underline{D}_{n}\right]=\left[\underline{L}_{D_{n}}\right]^{2}$ and $\left[\underline{K}_{n}\right]=\left[\underline{L}_{K_{n}}\right]^{2}$. Assuming that no available information (objective data) exits concerning the statistical dependence of the random generalized mass, damping and stiffness matrices, then it can be proved [10] that the full random matrices $\left[\mathbf{G}_{M_{n}}\right],\left[\mathbf{G}_{D_{n}}\right]$ or $\left[\mathbf{G}_{K_{n}}\right]$ have to be considered as mutually independent. The dispersion of random matrices $\left[\mathbf{G}_{M_{n}}\right],\left[\mathbf{G}_{D_{n}}\right]$ and $\left[\mathbf{G}_{K_{n}}\right]$ are controlled by the positive real parameters $\delta_{M}$, $\delta_{D}$ and $\delta_{K}$ which are independent of dimension $n$ and which do not depend on frequency $\omega$. If $A_{n}$ denotes $M_{n}, D_{n}$ or $K_{n}$, then the dispersion parameter $\delta_{A}$ of random matrix $\left[\mathbf{A}_{n}\right]$ is defined by

$$
\delta_{A}=\left\{\frac{1}{n} E\left\{\left\|\left[\mathbf{G}_{A_{n}}\right]-\left[I_{n}\right]\right\|_{F}^{2}\right\}\right\}^{1 / 2}
$$

The probability distribution $P_{\left[\mathbf{G}_{A_{n}}\right]}$ of the random matrix $\left[\mathbf{G}_{A_{n}}\right]$ is defined by a probability density function $\left[G_{n}\right] \mapsto p_{\left[\mathbf{G}_{A_{n}}\right]}\left(\left[G_{n}\right]\right)$ from $\mathbb{M}_{n}^{+}(\mathbb{R})$ into $\mathbb{R}^{+}=[0,+\infty[$, with respect to the measure $\widetilde{d} G_{n}$ on $\mathbb{M}_{n}^{S}(\mathbb{R})$, such that, $\widetilde{d} G_{n}=2^{n(n-1) / 4} \Pi_{1 \leq i \leq j \leq n} d\left[G_{n}\right]_{i j}$. We then have 
$P_{\left[\mathbf{G}_{A_{n}}\right]}=p_{\left[\mathbf{G}_{A_{n}}\right]}\left(\left[G_{n}\right]\right) \widetilde{d} G_{n}$ with the normalization condition $\int_{\mathbb{M}_{n}^{+}(\mathbb{R})} p_{\left[\mathbf{G}_{A_{n}}\right]}\left(\left[G_{n}\right]\right) \widetilde{d} G_{n}=1$.

The probability density function $p_{\left[\mathbf{G}_{A_{n}}\right]}\left(\left[G_{n}\right]\right)$ is then written $[10,11,12,20]$ as

$p_{\left[\mathbf{G}_{A_{n}}\right]}\left(\left[G_{n}\right]\right)=\mathbb{1}_{\mathbb{M}_{n}^{+}(\mathbb{R})}\left(\left[G_{n}\right]\right) \times C_{\mathbf{G}_{A_{n}}} \times\left(\operatorname{det}\left[G_{n}\right]\right)^{(n+1) \frac{\left(1-\delta_{A}^{2}\right)}{2 \delta_{A}^{2}}} \times \exp \left\{-\frac{(n+1)}{2 \delta_{A}^{2}} \operatorname{tr}\left[G_{n}\right]\right\}$,

in which $\mathbb{1}_{M_{n}^{+}(\mathbb{R})}\left(\left[G_{n}\right]\right)$ is equal to 1 if $\left[G_{n}\right] \in \mathbb{M}_{n}^{+}(\mathbb{R})$ and is equal to zero if $\left[G_{n}\right] \notin \mathbb{M}_{n}^{+}(\mathbb{R})$ and where the positive constant $C_{\mathbf{G}_{A_{n}}}$ is such that

$$
C_{\mathbf{G}_{A_{n}}}=\frac{(2 \pi)^{-n(n-1) / 4}\left(\frac{n+1}{2 \delta_{A}^{2}}\right)^{n(n+1)\left(2 \delta_{A}^{2}\right)^{-1}}}{\left\{\Pi_{j=1}^{n} \Gamma\left(\frac{n+1}{2 \delta_{A}^{2}}+\frac{1-j}{2}\right)\right\}}
$$

whith $\Gamma(z)$ the gamma function defined for $z>0$ by $\Gamma(z)=\int_{0}^{+\infty} t^{z-1} e^{-t} d t$. The above equation shows that $\left\{\left[\mathbf{G}_{A_{n}}\right]_{j k}, 1 \leq j \leq k \leq n\right\}$ are dependent random variables. In general, $(n+1) / \delta^{2}$ is not an integer and consequently, the probability distribution is not a Wishart distribution.

In order to solve the stochastic equation (6) by the Monte Carlo numerical simulation, it is necessary to construct a random matrix generator for $\left[\mathbf{G}_{A_{n}}\right]$ whose probability density function $p_{\left[\mathbf{G}_{A_{n}}\right]}\left(\left[G_{n}\right]\right)$ is defined above. The following algebraic representation developed in Refs. $[10,11,12,20]$ allows such a random matrix generator to be constructed. The random matrix $\left[\mathbf{G}_{A_{n}}\right]$, with dispersion parameter $\delta_{A}$ and having the probability density function $p_{\left[\mathbf{G}_{n}\right]}\left(\left[G_{n}\right]\right)$ defined above, can be written as

$$
\left[\mathbf{G}_{A_{n}}\right]=\left[\mathbf{L}_{A_{n}}\right]^{T}\left[\mathbf{L}_{A_{n}}\right],
$$

in which $\left[\mathbf{L}_{A_{n}}\right]$ is an upper triangular random $(n \times n)$ real matrix such that the random variables $\left\{\left[\mathbf{L}_{A_{n}}\right]_{j j^{\prime}}, j \leq j^{\prime}\right\}$ are mutually independent and such that

(1) for $j<j^{\prime}$, real-valued random variable $\left[\mathbf{L}_{A_{n}}\right]_{j j^{\prime}}$ is written as $\left[\mathbf{L}_{A_{n}}\right]_{j j^{\prime}}=\sigma_{n} U_{j j^{\prime}}$ in which $\sigma_{n}=\delta_{A}(n+1)^{-1 / 2}$ and where $U_{j j^{\prime}}$ is a real-valued Gaussian random variable with zero mean and variance equal to 1 ;

(2) for $j=j^{\prime}$, positive-valued random variable $\left[\mathbf{L}_{A_{n}}\right]_{j j}$ is written as $\left[\mathbf{L}_{A_{n}}\right]_{j j}=\sigma_{n} \sqrt{2 V_{j}}$ in which $\sigma_{n}$ is defined above and where $V_{j}$ is a positive-valued gamma random variable whose probability density function $p_{V_{j}}(v)$ with respect to $d v$ is written as $p_{V_{j}}(v)=\mathbb{1}_{\mathbb{R}^{+}}(v)\left\{\Gamma\left(\frac{n+1}{2 \delta_{A}^{2}}+\right.\right.$ $\left.\left.\frac{1-j}{2}\right)\right\}^{-1} v_{A}^{\frac{n+1}{2 \delta^{2}}-\frac{1+j}{2}} e^{-v}$. 


\section{Experimental estimation of the dispersion parameters for the non-parametric probabilistic model}

Let $\delta_{M}, \delta_{D}$ and $\delta_{K}$ be the dispersion parameters of the random generalized mass, damping and stiffness matrices. Since the dispersion parameters have to be independent of $n$ (see Section 6), the dispersion parameters can be estimated by using the experimental matrices $\left[M_{\nu}^{\exp }\left(\theta_{r}\right)\right],\left[D_{\nu}^{\exp }\left(\theta_{r}\right)\right]$ and $\left[K_{\nu}^{\exp }\left(\theta_{r}\right)\right]$ for $r=1, \ldots, 8$ corresponding to the 8 experimental sandwich panels, and for a dimension $\nu<n$. Here, a very simple procedure is proposed for estimating $\delta_{M}, \delta_{D}$ and $\delta_{K}$ (this procedure corresponds to the first step of the procedure based on the maximum likelihood principle and developed in Ref. [20]). The first step of this procedure consists in associating the $\nu$ first elastic modes computed with the updated mean finite element model, with the corresponding $\nu$ experimental elastic modes obtained by performing the experimental modal analysis $[15,16]$ of each sandwich panel. Let $0<$ $\omega_{j_{1}}^{\exp }\left(\theta_{r}\right) \leq \ldots \leq \omega_{j_{\nu}}^{\exp }\left(\theta_{r}\right)$ be the set of the $\nu$ experimental eigenfrequencies of sandwich panel $r$, corresponding to the set of the $\nu$ first eigenfrequencies $0<\underline{\omega}_{1} \leq \ldots \leq \underline{\omega}_{\nu}$ computed with the updated mean finite element model. The same set of degrees of freedom for the mean finite element model and for the experimental sandwich panels is considered (25 observations). For each sandwich panel $r=1, \ldots, 8$, the association of the first experimental elastic modes ordered in increasing eigenfrequencies (which means that $j_{1}=1, \ldots, j_{\nu}=\nu$ ), with the first elastic modes computed with the updated mean finite element model and ordered in increasing eigenfrequencies, is performed using the $\left[\operatorname{MAC}\left(\theta_{r}\right)\right]$ matrix defined by

$$
\left[\operatorname{MAC}\left(\theta_{r}\right)\right]_{\alpha \beta}=\frac{<\underline{\varphi}_{\beta}, \psi_{\alpha}^{\exp }\left(\theta_{r}\right)>^{2}}{<\underline{\varphi}_{\beta}, \underline{\varphi}_{\beta}><\psi_{\alpha}^{\exp }\left(\theta_{r}\right), \psi_{\alpha}^{\exp }\left(\theta_{r}\right)>}
$$

in which $\underline{\varphi}_{\beta}$ is the elastic mode of the updated mean finite element model whose eigenfrequency is $\underline{\omega}_{\beta}$ and where $\psi_{\alpha}^{\exp }\left(\theta_{r}\right)$ is the experimental elastic mode of sandwich panel $r$ whose eigenfrequeny is $\omega_{\alpha}^{\exp }\left(\theta_{r}\right)$. Let us consider the eight first elastic modes, i.e $\nu=8$. Let $[\overline{\mathrm{MAC}}]$ be the $(8 \times 8)$ real matrix corresponding to the average over the 8 panels and defined by $[\overline{\mathrm{MAC}}]=\frac{1}{8} \sum_{r=1}^{8}\left[\mathrm{MAC}\left(\theta_{r}\right)\right]$. The computation of this average MAC matrix yields

$$
[\overline{\mathrm{MAC}}]=\left[\begin{array}{llllllll}
\mathbf{0 . 9 6 7 7} & 0.0018 & 0.0594 & 0.0299 & 0.0045 & 0.0120 & 0.0018 & 0.0313 \\
0.0004 & \mathbf{0 . 9 6 0 0} & 0.0098 & 0.0284 & 0.0029 & 0.0387 & 0.0625 & 0.0006 \\
0.0508 & 0.0139 & \mathbf{0 . 9 6 0 6} & 0.0027 & 0.0035 & 0.0058 & 0.0490 & 0.0230 \\
0.0198 & 0.0105 & 0.0019 & \mathbf{0 . 9 7 6 1} & 0.0079 & 0.0040 & 0.0270 & 0.0301 \\
0.0129 & 0.0010 & 0.0012 & 0.0005 & \mathbf{0 . 9 7 7 5} & 0.0040 & 0.0270 & 0.0301 \\
0.0006 & 0.0302 & 0.0341 & 0.0000 & 0.0121 & \mathbf{0 . 9 1 2 4} & 0.0055 & 0.0068 \\
0.0010 & 0.0774 & 0.0135 & 0.0547 & 0.0006 & 0.0077 & \mathbf{0 . 9 1 7 7} & 0.0002 \\
0.0862 & 0.0002 & 0.0070 & 0.0232 & 0.0123 & 0.0079 & 0.0199 & \mathbf{0 . 8 0 5 3}
\end{array}\right]
$$


The matrix $[\overline{\mathrm{MAC}}]$ allows the optimal number $\nu$ to be defined (number of experimental elastic modes which can be associated with elastic modes computed with the updated mean finite element model). Fixing an error less than $4 \%$, this matrix shows that the diagonal terms are dominant and larger or equal to 0.96 for $\nu=5$. The optimal value is then $\nu=5$.

One has now to estimate the dispersion parameters of the three random matrices using $\nu=5$. Let $\left[\Psi_{\nu}^{\exp }\left(\theta_{r}\right)\right]$ be the $(m \times \nu)$ real matrix whose columns are the $\nu$ elastic modes of experimental sandwich panel $r$ associated with the first experimental eigenfrequencies $0<\omega_{1}^{\exp }\left(\theta_{r}\right) \leq \ldots \leq \omega_{\nu}^{\exp }\left(\theta_{r}\right)$ and let $\left[\underline{\Phi}_{\nu}\right]$ be the $(m \times \nu)$ real matrix whose columns are the $\nu$ first elastic modes calculated with the updated mean finite element model and associated with eigenfrequencies $0<\underline{\omega}_{1} \leq \ldots \leq \underline{\omega}_{\nu}$. Let $\left[\widetilde{M}_{\nu}^{\exp }\left(\theta_{r}\right)\right],\left[\widetilde{D}_{\nu}^{\exp }\left(\theta_{r}\right)\right]$ and $\left[\widetilde{K}_{\nu}^{\exp }\left(\theta_{r}\right)\right]$ be the corresponding experimental generalized mass, damping and stiffness matrices of experimental sandwich panel $r$ directly deduced from the experimental modal analysis and such that $\left[\widetilde{M}_{\nu}^{\exp }\left(\theta_{r}\right)\right]_{\alpha \alpha^{\prime}}=\mu_{\alpha}^{\exp }\left(\theta_{r}\right) \delta_{\alpha \alpha^{\prime}},\left[\widetilde{D}_{\nu}^{\exp }\left(\theta_{r}\right)\right]_{\alpha \alpha^{\prime}}=2 \xi_{\alpha}^{\exp }\left(\theta_{r}\right) \mu_{\alpha}^{\exp }\left(\theta_{r}\right) \omega_{\alpha}^{\exp }\left(\theta_{r}\right) \delta_{\alpha \alpha^{\prime}}$ and $\left[\widetilde{K}_{\nu}^{\exp }\left(\theta_{r}\right)\right]_{\alpha \alpha^{\prime}}=\mu_{\alpha}^{\exp }\left(\theta_{r}\right)\left(\omega_{\alpha}^{\exp }\left(\theta_{r}\right)\right)^{2} \delta_{\alpha \alpha^{\prime}}$. Let $\left[\mathbf{M}_{\nu}\right],\left[\mathbf{D}_{\nu}\right]$ and $\left[\mathbf{K}_{\nu}\right]$ be the random matrices associated with the mean reduced matrix model of dimension $\nu$ and defined in Section 6. Since the experimental elastic modes differ from the elastic modes constructed with the updated mean finite element model (due to uncertainties), matrices $\left[\widetilde{M}_{\nu}^{\exp }\left(\theta_{r}\right)\right],\left[\widetilde{D}_{\nu}^{\exp }\left(\theta_{r}\right)\right]$ and $\left[\widetilde{K}_{\nu}^{\exp }\left(\theta_{r}\right)\right]$ are not represented in the same vector subspace than $\left[\mathbf{M}_{\nu}\right],\left[\mathbf{D}_{\nu}\right]$ and $\left[\mathbf{K}_{\nu}\right]$ (or equivalently than $\left[\underline{M}_{\nu}\right],\left[\underline{D}_{\nu}\right]$ and $\left.\left[\underline{K}_{\nu}\right]\right)$. However, it can be written that

$$
\left[\Psi_{\nu}^{\exp }\left(\theta_{r}\right)\right] \widetilde{\mathbf{q}}^{\exp }\left(\theta_{r}\right)=\left[\underline{\Phi}_{\nu}\right] \mathbf{q}^{\exp }\left(\theta_{r}\right)
$$

in which $\widetilde{\mathbf{q}}^{\exp }\left(\theta_{r}\right)$ is the $\mathbb{C}^{m}$-vector of the experimental generalized coordinates and where $\mathbf{q}^{\exp }\left(\theta_{r}\right)$ is the corresponding $\mathbb{C}^{m}$-vector of the generalized coordinates in the mean-model basis. By construction, the matrix $\left[\Psi_{\nu}^{\exp }\left(\theta_{r}\right)\right]^{T}\left[\Psi_{\nu}^{\exp }\left(\theta_{r}\right)\right] \in \mathbb{M}_{\nu}(\mathbb{R})$ is invertible. Introducing the left pseudo-inverse $\left(\left[\Psi_{\nu}^{\exp }\left(\theta_{r}\right)\right]^{T}\left[\Psi_{\nu}^{\exp }\left(\theta_{r}\right)\right]\right)^{-1}\left[\Psi_{\nu}^{\exp }\left(\theta_{r}\right)\right]^{T} \in \mathbb{M}_{\nu, m}(\mathbb{R})$ of $\left[\Psi_{\nu}^{\exp }\left(\theta_{r}\right)\right] \in$ $M_{m, \nu}(\mathbb{R})$, Eq. (12) yields

$$
\widetilde{\mathbf{q}}^{\exp }\left(\theta_{r}\right)=\left[S_{\nu}^{\exp }\left(\theta_{r}\right)\right] \mathbf{q}^{\exp }\left(\theta_{r}\right)
$$

in which the matrix $\left[S_{\nu}^{\exp }\left(\theta_{r}\right)\right] \in \mathbb{M}_{\nu}(\mathbb{R})$ is written as

$$
\left[S_{\nu}^{\exp }\left(\theta_{r}\right)\right]=\left(\left[\Psi_{\nu}^{\exp }\left(\theta_{r}\right)\right]^{T}\left[\Psi_{\nu}^{\exp }\left(\theta_{r}\right)\right]\right)^{-1}\left[\Psi_{\nu}^{\exp }\left(\theta_{r}\right)\right]^{T}\left[\underline{\Phi}_{\nu}\right]
$$

The matrix transformation defined by Eqs. (13)-(14) allows the experimental matrices $\left[\widetilde{M}_{\nu}^{\exp }\left(\theta_{r}\right)\right],\left[\widetilde{D}_{\nu}^{\exp }\left(\theta_{r}\right)\right]$ and $\left[\widetilde{K}_{\nu}^{\exp }\left(\theta_{r}\right)\right]$ to be transformed into the matrices $\left[M_{\nu}^{\exp }\left(\theta_{r}\right)\right]$, 
$\left[D_{\nu}^{\exp }\left(\theta_{r}\right)\right]$ and $\left[K_{\nu}^{\exp }\left(\theta_{r}\right)\right]$, which are defined by

$$
\begin{aligned}
& {\left[M_{\nu}^{\exp }\left(\theta_{r}\right)\right]=\left[S_{\nu}^{\exp }\left(\theta_{r}\right)\right]^{T}\left[\widetilde{M}_{\nu}^{\exp }\left(\theta_{r}\right)\right]\left[S_{\nu}^{\exp }\left(\theta_{r}\right)\right] \in \mathbb{M}_{\nu}^{+}(\mathbb{R})} \\
& {\left[D_{\nu}^{\exp }\left(\theta_{r}\right)\right]=\left[S_{\nu}^{\exp }\left(\theta_{r}\right)\right]^{T}\left[\widetilde{D}_{\nu}^{\exp }\left(\theta_{r}\right)\right]\left[S_{\nu}^{\exp }\left(\theta_{r}\right)\right] \in \mathbb{M}_{\nu}^{+}(\mathbb{R})} \\
& {\left[K_{\nu}^{\exp }\left(\theta_{r}\right)\right]=\left[S_{\nu}^{\exp }\left(\theta_{r}\right)\right]^{T}\left[\widetilde{K}_{\nu}^{\exp }\left(\theta_{r}\right)\right]\left[S_{\nu}^{\exp }\left(\theta_{r}\right)\right] \in \mathbb{M}_{\nu}^{+}(\mathbb{R})}
\end{aligned}
$$

Let $A$ be $M, D$ or $K$. One can then introduce the matrix $\left[G_{A_{\nu}}^{\exp }\left(\theta_{r}\right)\right] \in \mathbb{M}_{n}^{+}(\mathbb{R})$ such that $\left[A_{\nu}^{\exp }\left(\theta_{r}\right)\right]=\left[\underline{L}_{A_{\nu}}\right]^{T}\left[G_{A_{\nu}}^{\exp }\left(\theta_{r}\right)\right]\left[\underline{L}_{A_{\nu}}\right]$ in which the invertible upper triangular matrix $\left[\underline{L}_{A_{\nu}}\right] \in$ $\mathbb{M}_{\nu}(\mathbb{R})$ is such that $\left[\underline{A}_{\nu}\right]=\left[\underline{L}_{A_{\nu}}\right]^{T}\left[\underline{L}_{A_{\nu}}\right] \in \mathbb{M}_{n}^{+}(\mathbb{R})$. Therefore, matrix $\left[G_{A_{\nu}}^{\exp }\left(\theta_{r}\right)\right]$ is given by the equation,

$$
\left[G_{A_{\nu}}^{\exp }\left(\theta_{r}\right)\right]=\left[\underline{L}_{A_{\nu}}\right]^{-T}\left[A_{\nu}^{\exp }\left(\theta_{r}\right)\right]\left[\underline{L}_{A_{\nu}}\right]^{-1} \in \mathbb{M}_{\nu}^{+}(\mathbb{R})
$$

Consequently, the eight realizations $\left\{\left[G_{A_{\nu}}^{\exp }\left(\theta_{r}\right)\right], r=1, \ldots, 8\right\}$ of random matrix $\left[\mathbf{G}_{A_{\nu}}\right]$ defined by Eq. (11) have effectively been constructed. The dispersion parameter $\delta_{A}$ of random matrix $\left[\mathbf{A}_{\nu}\right]$, defined by Eq. (10) for $n=\nu=5$, has to be chosen independent of $n$ and is then estimated by

$$
\delta_{A}=\left\{\frac{1}{8 \nu} \sum_{r=1}^{8}\left\|\left[G_{A_{\nu}}^{\exp }\left(\theta_{r}\right)\right]-\left[I_{\nu}\right]\right\|_{F}^{2}\right\}^{1 / 2} .
$$

From Eq. (17), it can be deduced that $\delta_{M}=0.23, \delta_{D}=0.43$ and $\delta_{K}=0.25$. Consequently, these values represent the dispersion parameters for random matrices $\left[\mathbf{M}_{n}\right],\left[\mathbf{D}_{n}\right]$ and $\left[\mathbf{K}_{n}\right]$. These dispersion parameters are taken as constants independent of dimension $n$.

Since the number of experimental panels is relatively small ( 8 panels are used), the quality of the estimation of $\delta_{A}$ defined by Eq. (17) could be questionnable. Nevertheless, as explain below, such an estimation is perfectly correct. In Ref. [20], the convergence of the estimator $\widehat{\delta}_{A}$ used to calculate the estimation $\delta_{A}$ defined by Eq. (17) has been studied and one reuses this result. Applying this result for a dimension $\nu=5$ of the random matrix $\left[G_{A_{\nu}}^{\exp }\right]$ and for 8 realizations, yields a standard deviation $\sigma_{\widehat{\delta}_{A}}$ of the estimator $\widehat{\delta}_{A}$ which is equal to 0.0146 for $\delta_{M}=0.23$, to 0.0328 for $\delta_{D}=0.43$ and to 0.0171 for $\delta_{K}=0.25$. Consequently, although the number of realizations is relatively small ( 8 panels), the estimator is reasonably converged and then, the estimation can be considered as correct. This unexpected and unusual result is due to the struture of the random matrix $\left[G_{A_{\nu}}^{\exp }\right]$. As it can be seen in Eq. (11), for $n=\nu$, this random matrix depends on $\nu \times(\nu+1) / 2$ independent random variables, i.e, on 15 independent random variables. Consequently, each realization of this random matrix is spanned by the realizations of 15 independent random variables, and therefore, the estimation $\delta_{A}$ is performed by using $8 \times 15=120$ realizations of independent random variables. In general, such an argument is 
wrong, but in the present case, due to the structure of the random matrix $\left[G_{A_{\nu}}^{\exp }\right]$ and due to Eq. (10) defining $\delta_{A}$, this result holds and has been proved in studying the standard deviation $\sigma_{\widehat{\delta}_{A}}$ of estimator $\widehat{\delta}_{A}$ (see Ref. [20]).

\section{Confidence region prediction for the FRF and experimental comparisons}

\subsection{Confidence region prediction with the non-parametric probabilistic model}

We are interested in the construction of the confidence region associated with a probability level $P_{c}=0.96$ for the modulus of the random cross frequency response functions between point $N 0$ and points $N 1, N 2$ and $N 3$. Let $\omega \mapsto W(\omega)=\left|-\omega^{2} \mathbf{Y}_{k}^{n}(\omega)\right|$ in which $k$ is the degree of freedom corresponding to the $z$-displacement at points $N 1, N 2$ and $N 3$, and where $\mathbf{Y}^{n}(\omega)$ is the random vector given by Eqs. (5) and (6). This confidence region is constructed by using the sample quantiles [20]. For $\omega$ fixed in $B$, let $F_{W(\omega)}$ be the cumulative distribution function (continuous from the right) of random variable $W(\omega)$ which is such that $F_{W(\omega)}(w)=P(W(\omega) \leq w)$. For $0<p<1$, the $p$ th quantile or fractile of $F_{W(\omega)}$ is defined as

$$
\zeta(p)=\inf \left\{w: F_{W(\omega)}(w) \geq p\right\}
$$

Then, the upper envelope $w^{+}(\omega)$ and the lower envelope $w^{-}(\omega)$ of the confidence region are defined by

$$
w^{+}(\omega)=\zeta\left(\left(1+P_{c}\right) / 2\right) \quad, \quad w^{-}(\omega)=\zeta\left(\left(1-P_{c}\right) / 2\right)
$$

The estimation of $w^{+}(\omega)$ and $w^{-}(\omega)$ is performed as follows. Let $w_{1}(\omega)=W\left(\omega ; \theta_{1}\right), \ldots$, $w_{n_{s}}(\omega)=W\left(\omega ; \theta_{n_{s}}\right)$ be the $n_{s}$ independent realizations of random variable $W(\omega)$ associated with the independent realizations $\theta_{1}, \ldots, \theta_{n_{s}}$. Let $\widetilde{w}_{1}(\omega)<\ldots<\widetilde{w}_{n_{s}}(\omega)$ be the order statistics associated with $w_{1}(\omega), \ldots, w_{n_{s}}(\omega)$. Therefore, one has the following estimation

$$
\begin{aligned}
& w^{+}(\omega) \simeq \widetilde{w}_{j^{+}}(\omega) \quad, \quad j^{+}=\operatorname{fix}\left(n_{s}\left(1+P_{c}\right) / 2\right) \\
& w^{-}(\omega) \simeq \widetilde{w}_{j^{-}}(\omega), \quad, \quad j^{-}=\operatorname{fix}\left(n_{s}\left(1-P_{c}\right) / 2\right)
\end{aligned}
$$

in which $\operatorname{fix}(z)$ is the integer part of the real number $z$.

The confidence region of the random cross frequency response functions are calculated by using Eqs. (5)-(11) and (20)-(21). Random Eqs. (5) and (6) are solved by using the Monte Carlo numerical simulation with $n_{s}$ realizations. The realization $\mathbf{Q}^{n}\left(\omega ; a_{\ell}\right)$ of the $\mathbb{C}^{n}$-valued random variable $\mathbf{Q}^{n}(\omega)$ is the solution of the deterministic matrix equation

$$
\left(-\omega^{2}\left[\mathbf{M}_{n}\left(a_{\ell}\right)\right]+i \omega\left[\mathbf{D}_{n}\left(a_{\ell}\right)\right]+\left[\mathbf{K}_{n}\left(a_{\ell}\right)\right]\right) \mathbf{Q}^{n}\left(\omega ; a_{\ell}\right)=\underline{\mathcal{F}}^{n}(\omega) \quad, \quad \omega \in B
$$


in which $\left[\mathbf{M}_{n}\left(a_{\ell}\right)\right],\left[\mathbf{D}_{n}\left(a_{\ell}\right)\right]$ and $\left[\mathbf{K}_{n}\left(a_{\ell}\right)\right]$ are the realizations of the random matrices $\left[\mathbf{M}_{n}\right]$, $\left[\mathbf{D}_{n}\right]$ and $\left[\mathbf{K}_{n}\right]$ respectively. The convergence of the random solution of Eq. (6) with respect to the number $n_{s}$ of realizations can be analyzed in studying the mapping

$$
n_{s} \mapsto \operatorname{Conv}\left(n_{s}\right)=\frac{1}{n_{s}} \sum_{\ell=1}^{n_{s}} \int_{B}\left\|\mathbf{Q}^{n}\left(\omega ; a_{\ell}\right)\right\|^{2} d \omega
$$

in which $\mathbf{Q}^{n}\left(\omega ; a_{1}\right), \ldots, \mathbf{Q}^{n}\left(\omega ; a_{n_{s}}\right)$ are the $n_{s}$ realizations of the $\mathbb{C}^{n}$-valued random variable $\mathbf{Q}^{n}(\omega)$. Figure 10 displays the graph of the function $n_{s} \mapsto \operatorname{Conv}\left(n_{s}\right)$ for $n=200$. The convergence is reached for $n_{s}=1200$.

\subsection{Prediction and experimental comparison}

Figures 11, 12 and 13 display the confidence region prediction for the random cross frequency response functions between point $N 0$ and points $N 1, N 2$ and $N 3$ respectively, calculated with $n_{s}=2000$ realizations and $n=200$. These figures show how the experimental cross FRF corresponding to the 8 panels are positioned with respect to this confidence region. In addition, each figure displays the graph of the numerical cross FRF calculated with the updated mean reduced matrix model and the graph of the mean value of the random cross FRF calculated with the non-parametric probabilistic model. It should be noted that the experimental responses belong almost always to the predicted confidence region but sometimes, do not belong to the confidence region. In particular, it is due to the fact that the predicted confidence region is calculated with a probability level 0.96 and not with the level 1! Consequently, these figures show that the prediction compared with the experiments is good.

\section{Conclusions}

The methodology proposed to experimentally indentify the non-parametric probabilistic approach which allows model and data uncertainties to be taken into account in structural dynamics has been validated. The experimental results obtained for a set of 8 light sandwich panels show the sensitivity of the dynamical response of the panels in the medium-frequency range. The use of the simplified usual laminated composite thin plate theory, for constructing the predictive dynamical mean model, introduces significant model uncertainties in the medium-frequency range. Since such dynamical systems are very sensitive to uncertainties and taking into account the presence of data and model uncertainties in the mean mechanical model, the introduction of a probabilistic model of model uncertainties is necessary to improve the predictability of the mean model in the medium-frequency range. The confidence regions of the cross frequency response functions of the stochastic systems are then constructed and are 
compared to the experimental cross frequency response functions for the 8 sandwich panels. The prediction compared with the experiments is good.

\section{References}

[1] L. P. Chao, M. V. Gandhi, B. S. Thompson, Design for manufacture methodology for incorporating uncertainties in the robust design of fibrous laminated composite structures, Journal of Composite Materials 27 (2) (1993) 175-194.

[2] G. Van Vinckenroy, W.P. de Wilde, The use of Monte Carlo techniques in statistical finite element methods for the determination of the structural behaviour of composite materials structural components, Composite Structures 32 (1995) 247-253.

[3] A. K. Noor, J. H. Starnes Jr., J. M. Peters, Uncertainty analysis of composite structures, Computer Methods in Applied Mechanics and Engineering 185 (2000) 413-432.

[4] A. K. Noor, J. H. Starnes Jr., J. M. Peters, Uncertainty analysis of stiffened composite panels, Computer \& Structures 51 (2001) 139-158.

[5] T.-U. Kim, H.-C. Sin, Optimal design of composite laminated plates with the discreteness in ply angles and uncertainty in material properties considered, Computer \& Structures 79 (2001) 2501-2509.

[6] M. Cho, S. Y. Rhee, Optimization of laminates with free edges under bounded uncertainty subject to extension, bending and twisting, Solids and Structures 41 (2004) 227-245.

[7] B. N. Singh, D. Yadav, N. G. R. Iyengar, Stability analysis of laminated cylindrical panels with uncertain material properties, Composite Structures 54 (2001) 17-26.

[8] D. H. Oh, L. Librescu, Free vibration and reliability of composite cantilevers featuring uncertain properties, Reliability Engineering and System Safety 56 (1997) 265-272.

[9] B. N. Singh, D. Yadav, N. G. R. Iyengar, Free vibration of composite cylindrical panels with random material properties, Composite Structures 58 (2002) 435-442.

[10] C. Soize, A nonparametric model of random uncertainties on reduced matrix model in structural dynamics, Probabilistic Engineering Mechanics 15 (3)(2000) 277-294.

[11] C. Soize, Maximum entropy approach for modeling random uncertainties in transient elastodynamics, Journal of the Acoustical Society of America 109 (5) (2001) 1979-1996.

[12] C. Soize, A comprehensive overview of a non-parametric probabilistic approach of model uncertainties for predictive models in structural dynamics, Journal of Sound and Vibration 288 (2005) 623-652.

[13] K. McConnell, Vibration Testing. Theory and Practice, Wiley Interscience, New York, 1995. 
[14] J. S. Bendat, A. G. Piersol, Engineering Applications of Correlation and Spectral Analysis, John Wiley And Sons, New York, 1980.

[15] D. Ewins, Modal Testing: Theory and Practice, John Wiley and Sons, Inc., New York, 1984.

[16] E. Balmes, Structural Dynamics Toolbox for Use with Matlab, Scientific Software, 2000.

[17] O.O. Ochoa, J.N. Reddy, Finite Element Analysis of Composite Laminates, Kluwer Academic Publishers, 1992.

[18] J.N. Reddy, Mechanics of Laminated Composite Plates, CRC Press, 1997.

[19] R.M. Jones, Mechanics of Composite Materials, Taylor and Francis, 1999.

[20] C. Soize, Random matrix theory for modeling uncertainties in computational mechanics, Computer Methods in Applied Mechanics and Engineering, 194(12-16), 1333-1366 (2005).

[21] R.J. Serfling, Approximation Theorems of Mathematical Statistics, John Wiley \& Sons, 1980. 


\section{Figure captions}

Fig.1: Step 2 of the manufacturing process of a sandwich panel

Figure 2: Graphs of the 8 experimental cross FRF between point N0 and point N1 corresponding to the 8 sandwich panels. Horizontal axis: frequency in Hertz. Vertical axis: $\log _{10}$ of the modulus of the acceleration in $\mathrm{m} / \mathrm{s}^{2}$.

Figure 3: Graphs of the 8 experimental cross FRF between point N0 and point N2 corresponding to the 8 sandwich panels. Horizontal axis: frequency in Hertz. Vertical axis: $\log _{10}$ of the modulus of the acceleration in $\mathrm{m} / \mathrm{s}^{2}$.

Figure 4: Graphs of the 8 experimental cross FRF between point N0 and point N3 corresponding to the 8 sandwich panels. Horizontal axis: frequency in Hertz. Vertical axis: $\log _{10}$ of the modulus of the acceleration in $\mathrm{m} / \mathrm{s}^{2}$.

Figure 5: Convergence of the cross frequency response function between point $N 0$ and point $N 1$ for three finite element meshes: $32 \times 32$ (thin solid line), $64 \times 64$ ( thick solid line), $128 \times 64$ (thin dashed line). Horizontal axis: frequency in Hertz. Vertical axis: $\log _{10}$ of the modulus of the acceleration in $\mathrm{m} / \mathrm{s}^{2}$.

Figure 6: Convergence of the $L^{2}$-norm in space and in frequency of $z$-acceleration response (vertical axis) versus the dimension of the updated mean reduced matrix model (horizontal axis).

Figure 7: Graphs of the cross FRF between point $N 0$ and point $N 1$. Horizontal axis: frequency in Hertz. Vertical axis: $\log _{10}$ of the modulus of the acceleration in $\mathrm{m} / \mathrm{s}^{2}$. Experimental cross FRF corresponding to the 8 panels ( 8 thin solid lines). Numerical cross FRF calulated with the updated mean reduced matrix model (thick solid line)

Figure 8: Graphs of the cross FRF between point $N 0$ and point $N 2$. Horizontal axis: frequency in Hertz. Vertical axis: $\log _{10}$ of the modulus of the acceleration in $\mathrm{m} / \mathrm{s}^{2}$. Experimental cross FRF corresponding to the 8 panels ( 8 thin solid lines). Numerical cross FRF calulated with the updated mean reduced matrix model (thick solid line)

Figure 9: Graphs of the cross FRF between point $N 0$ and point $N 3$. Horizontal axis: frequency in Hertz. Vertical axis: $\log _{10}$ of the modulus of the acceleration in $\mathrm{m} / \mathrm{s}^{2}$. Experimental cross FRF corresponding to the 8 panels ( 8 thin solid lines). Numerical cross FRF calulated with the updated mean reduced matrix model (thick solid line) 
Figure 10: Convergence of the random solution with respect to the number of realizations: Graph of function $n_{s} \mapsto \operatorname{Conv}\left(n_{s}\right)$. Horizontal axis: $n_{s}$. Vertical axis: $\operatorname{Conv}\left(n_{s}\right)$.

Figure 11: Confidence region prediction for the random cross FRF between point $N 0$ and point $N 1$. Horizontal axis: frequency in Hertz. Vertical axis: $\log _{10}$ of the modulus of the acceleration in $m / s^{2}$. Experimental cross FRF corresponding to the 8 panels ( 8 thin solid lines). Numerical cross FRF calculated with the updated mean reduced matrix model (thick solid line). Mean value of the random cross FRF calculated with the non-parametric probabilistic model (thin dashed line). Confidence region of the random cross FRF calculated with the non-parametric probabilistic model (grey region).

Figure 12: Confidence region prediction for the random cross FRF between point $N 0$ and point $N 2$. Horizontal axis: frequency in Hertz. Vertical axis: $\log _{10}$ of the modulus of the acceleration in $m / s^{2}$. Experimental cross FRF corresponding to the 8 panels ( 8 thin solid lines). Numerical cross FRF calculated with the updated mean reduced matrix model (thick solid line). Mean value of the random cross FRF calculated with the non-parametric probabilistic model (thin dashed line). Confidence region of the random cross FRF calculated with the non-parametric probabilistic model (grey region).

Figure 13: Confidence region prediction for the random cross FRF between point $N 0$ and point N3. Horizontal axis: frequency in Hertz. Vertical axis: $\log _{10}$ of the modulus of the acceleration in $m / s^{2}$. Experimental cross FRF corresponding to the 8 panels ( 8 thin solid lines). Numerical cross FRF calculated with the updated mean reduced matrix model (thick solid line). Mean value of the random cross FRF calculated with the non-parametric probabilistic model (thin dashed line). Confidence region of the random cross FRF calculated with the non-parametric probabilistic model (grey region). 


\section{FIGURES}

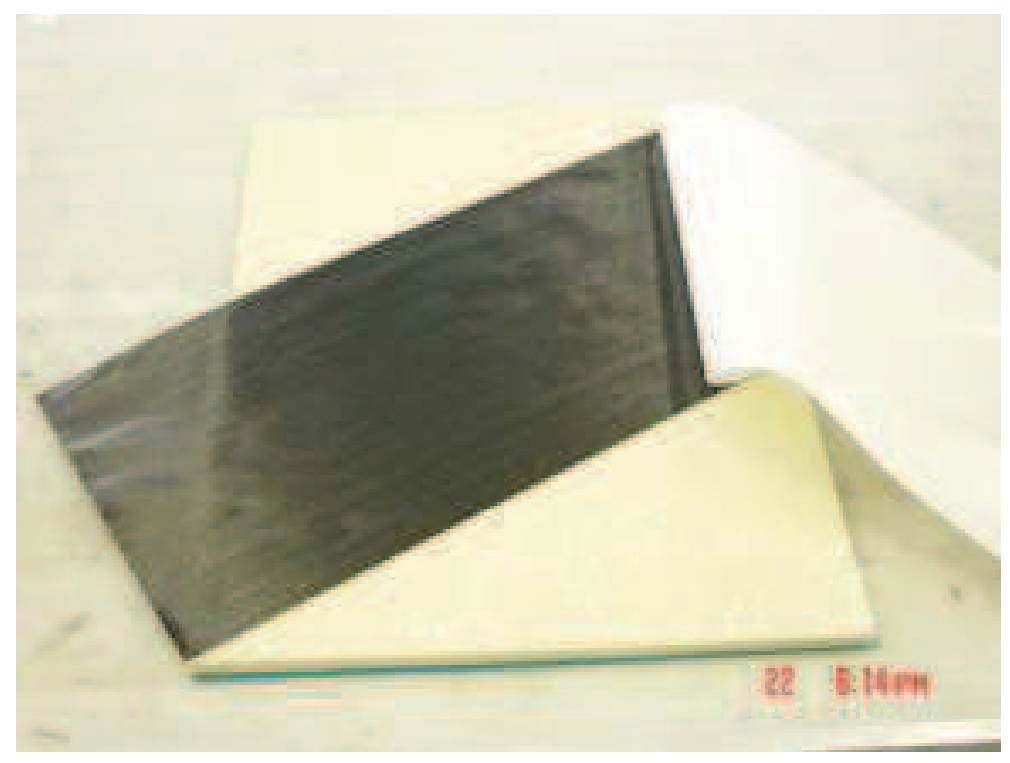

Figure 1

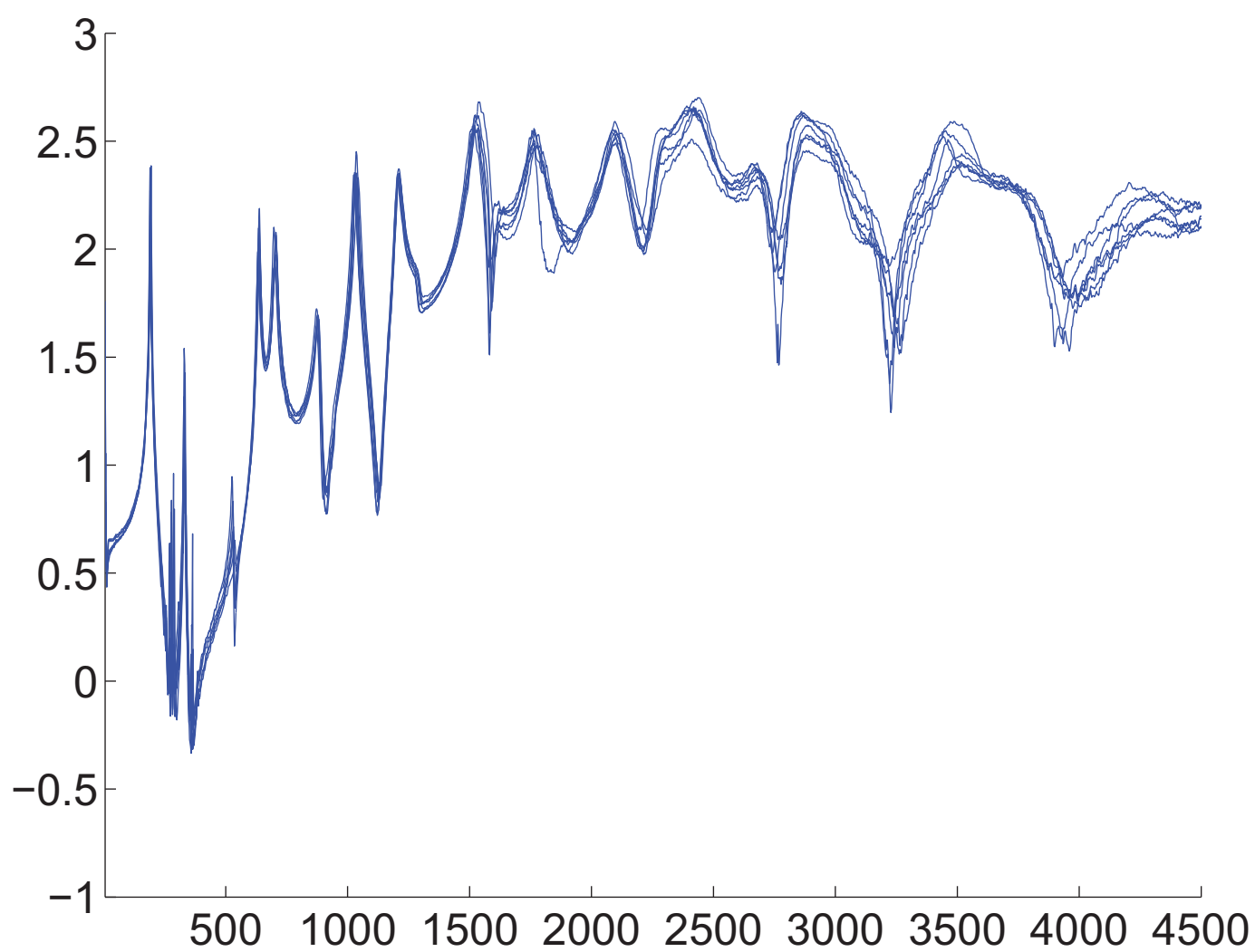

Figure 2 


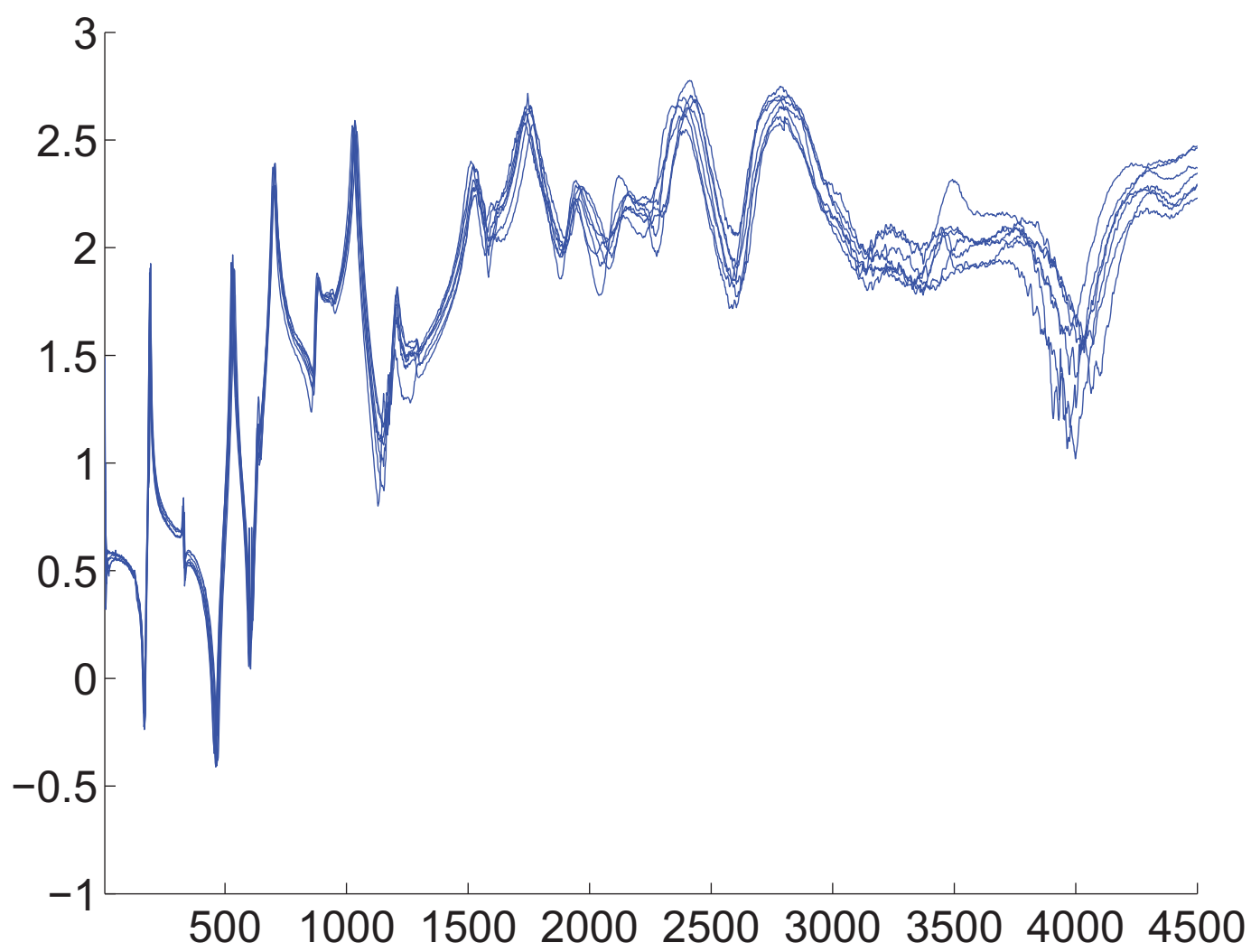

Figure 3

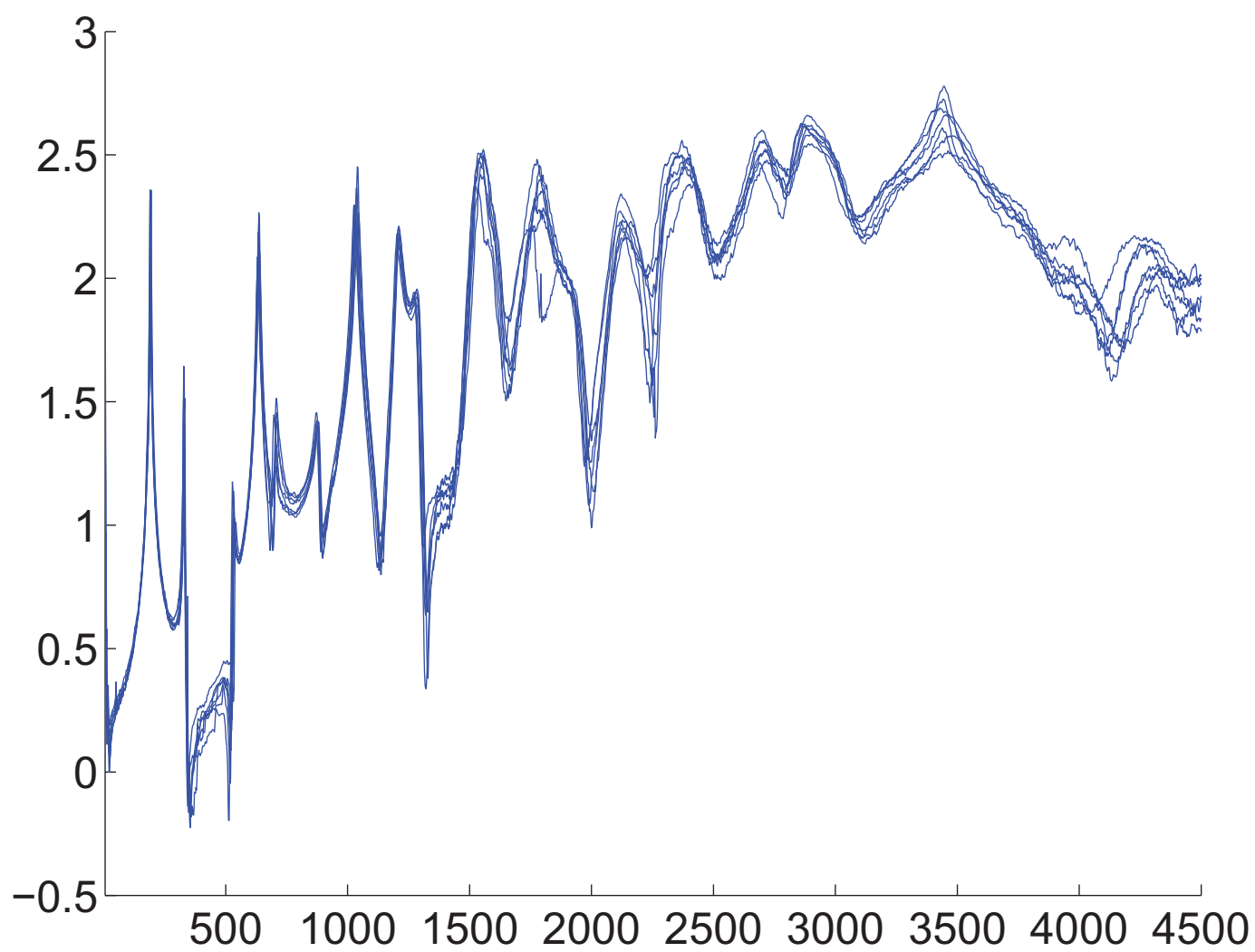

Figure 4 


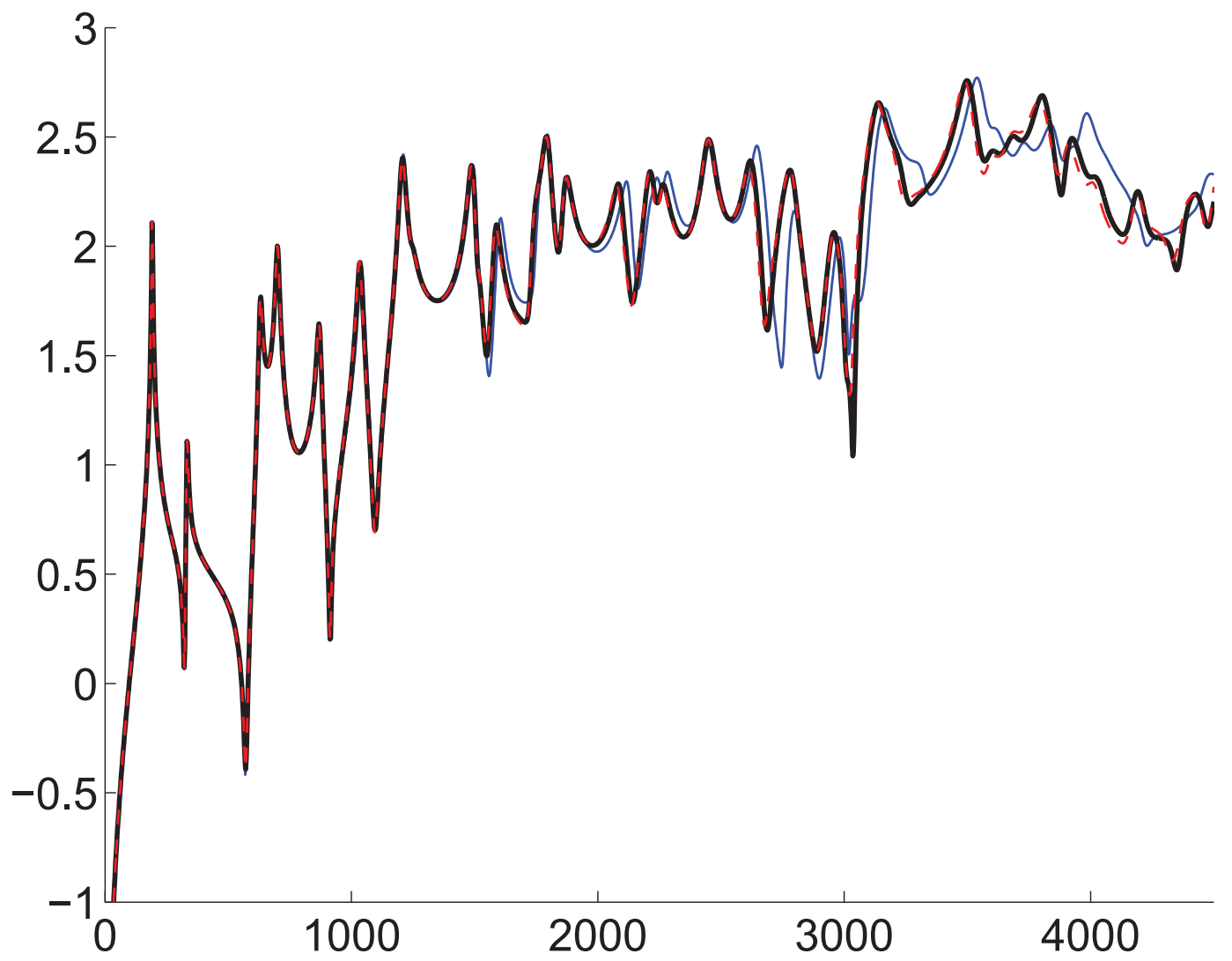

Figure 5

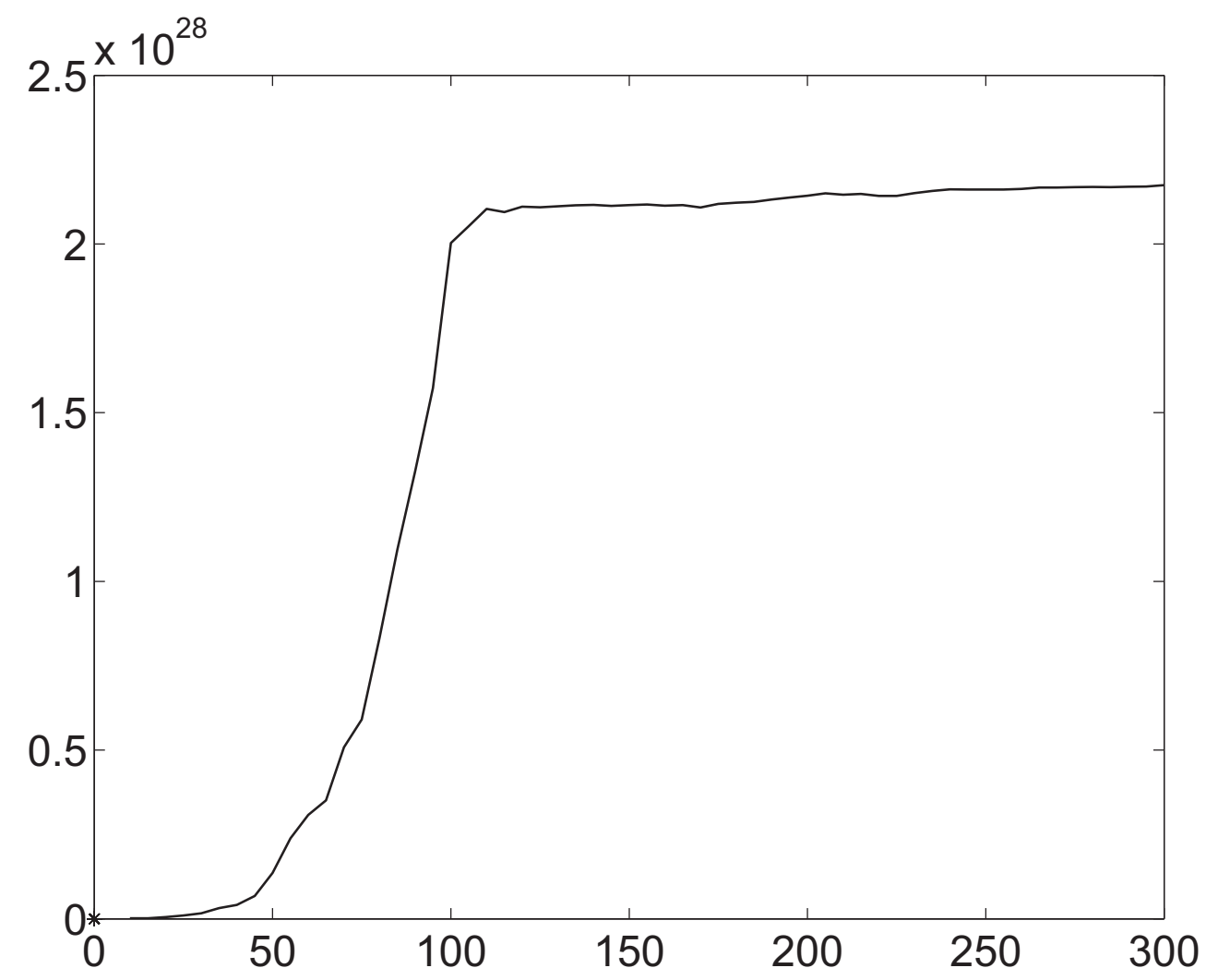

Figure 6 


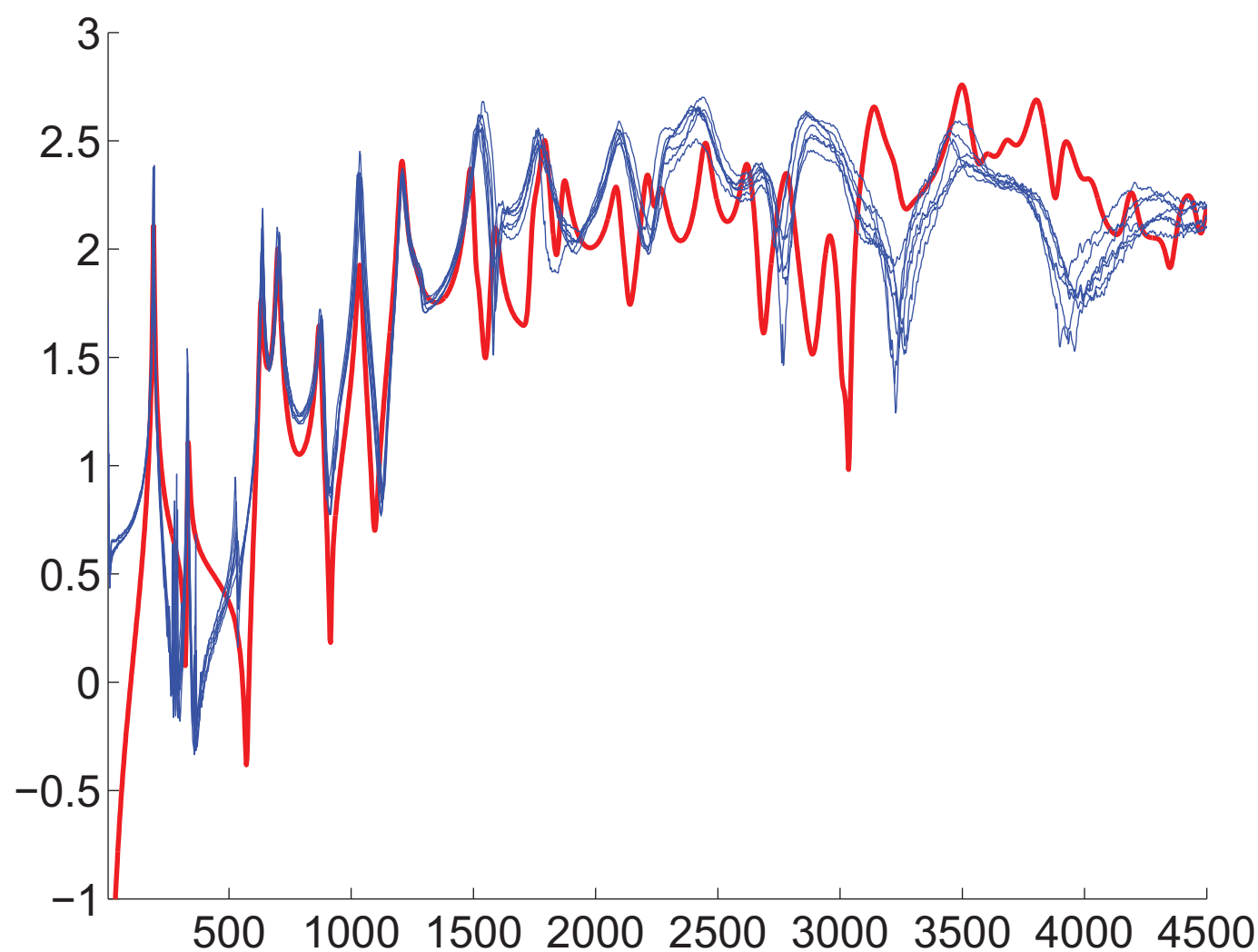

Figure 7

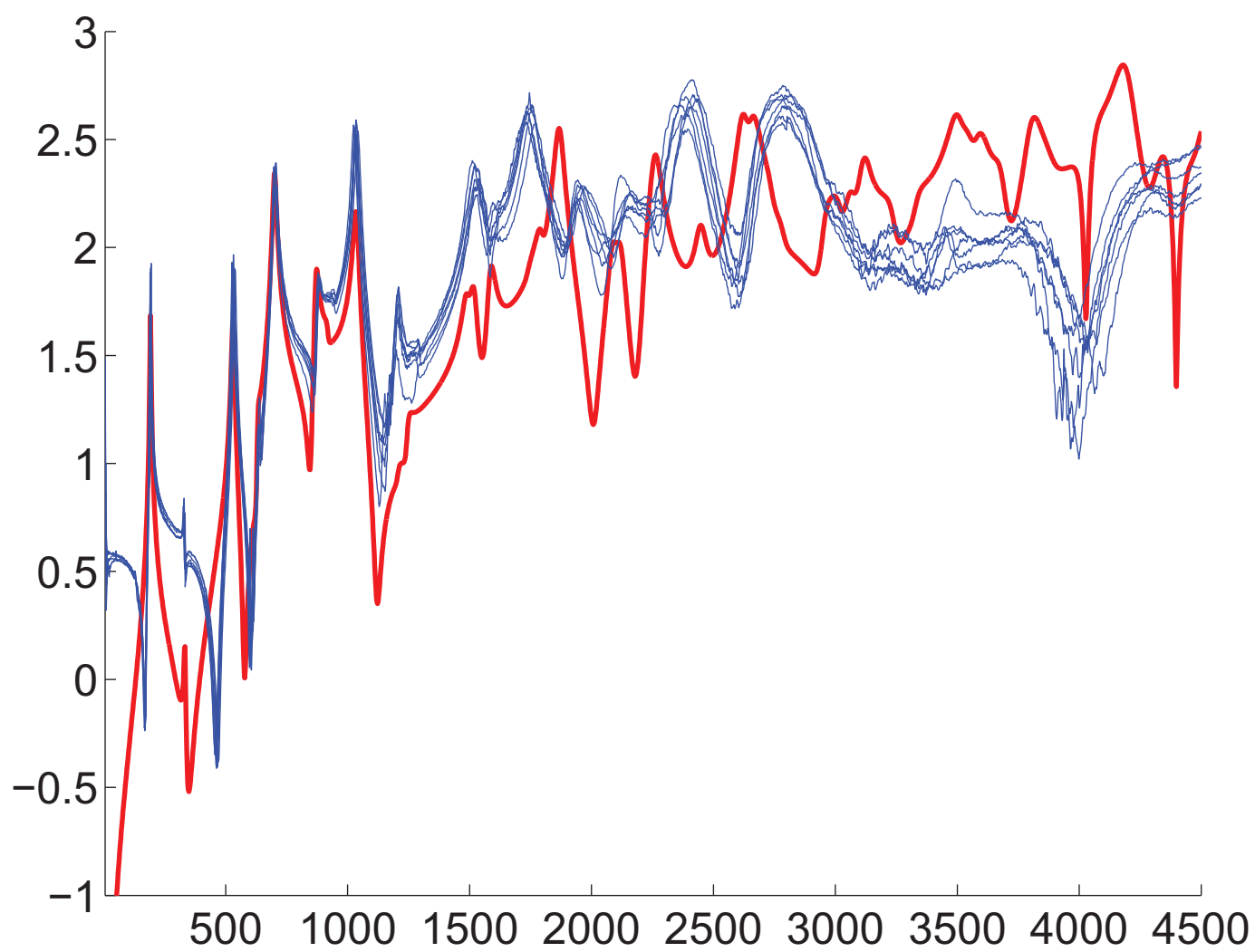

Figure 8 


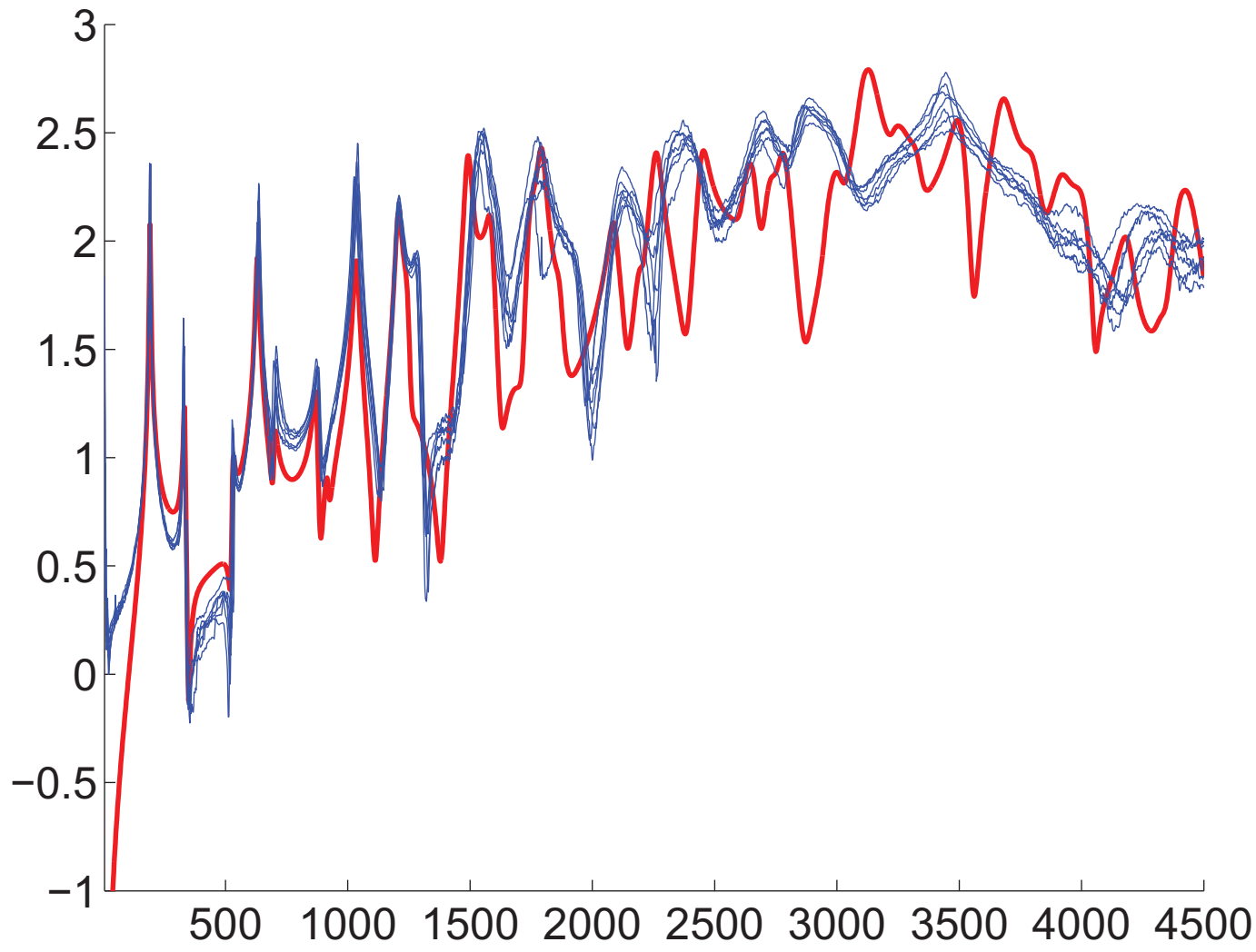

Figure 9

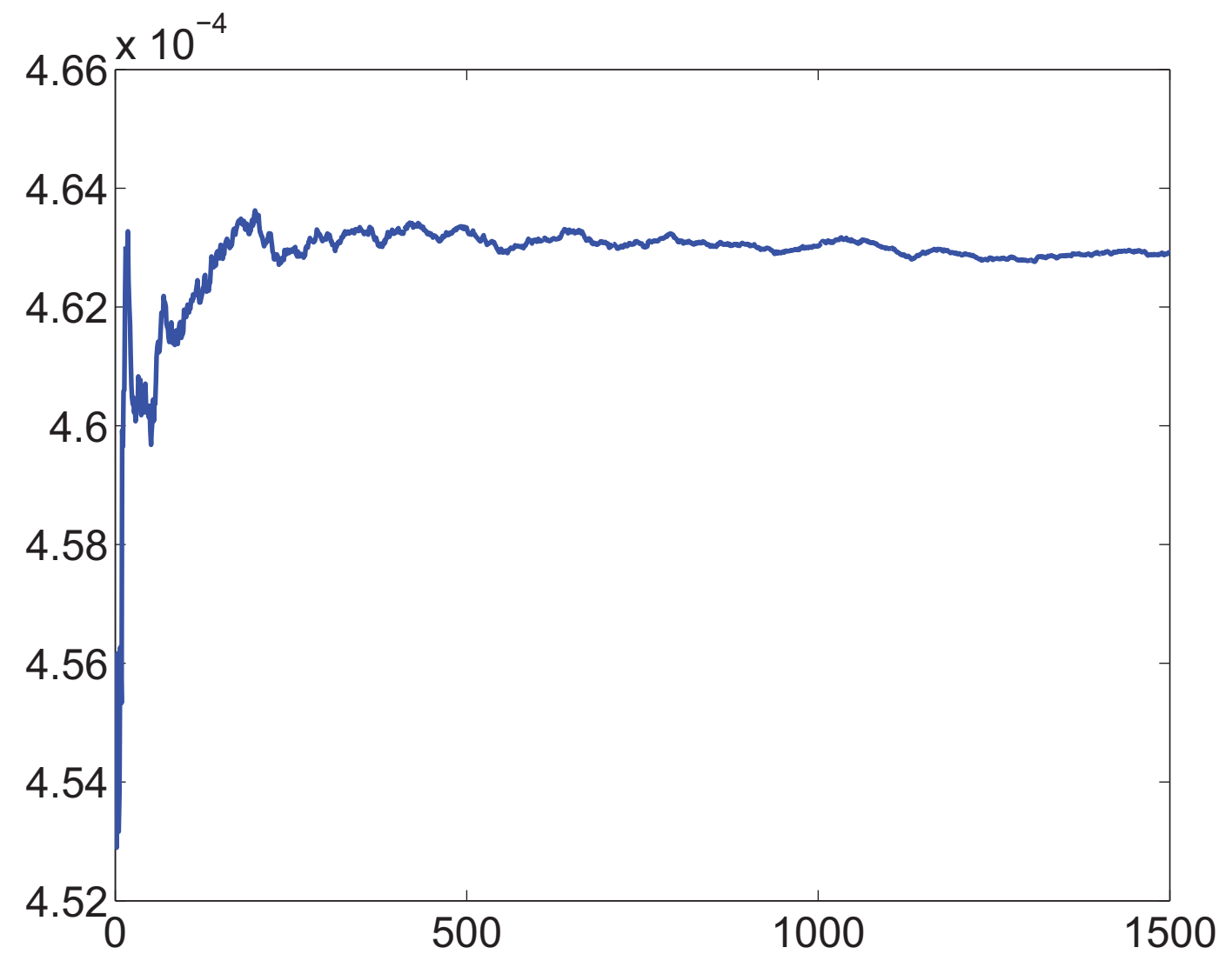

Figure 10 


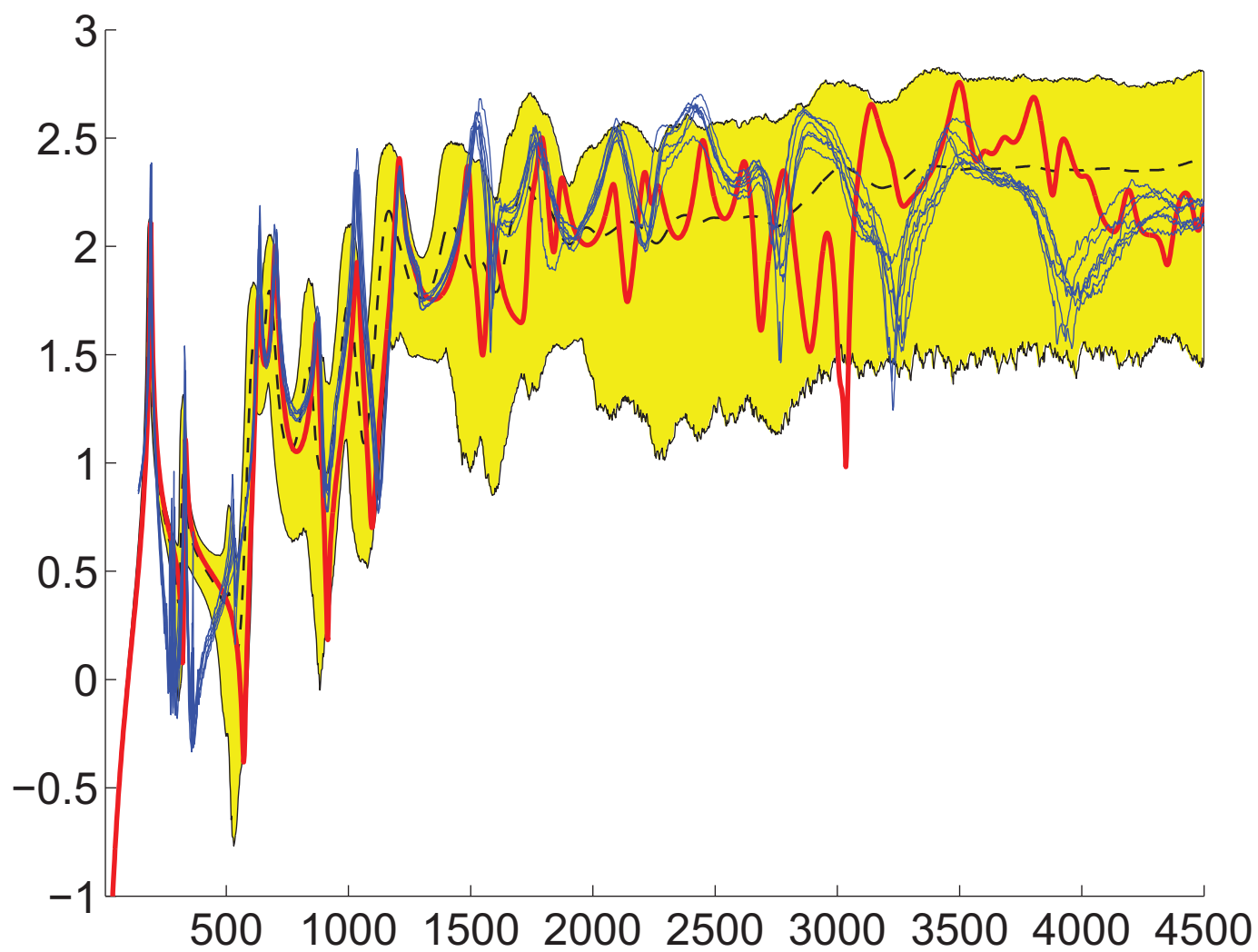

Figure 11

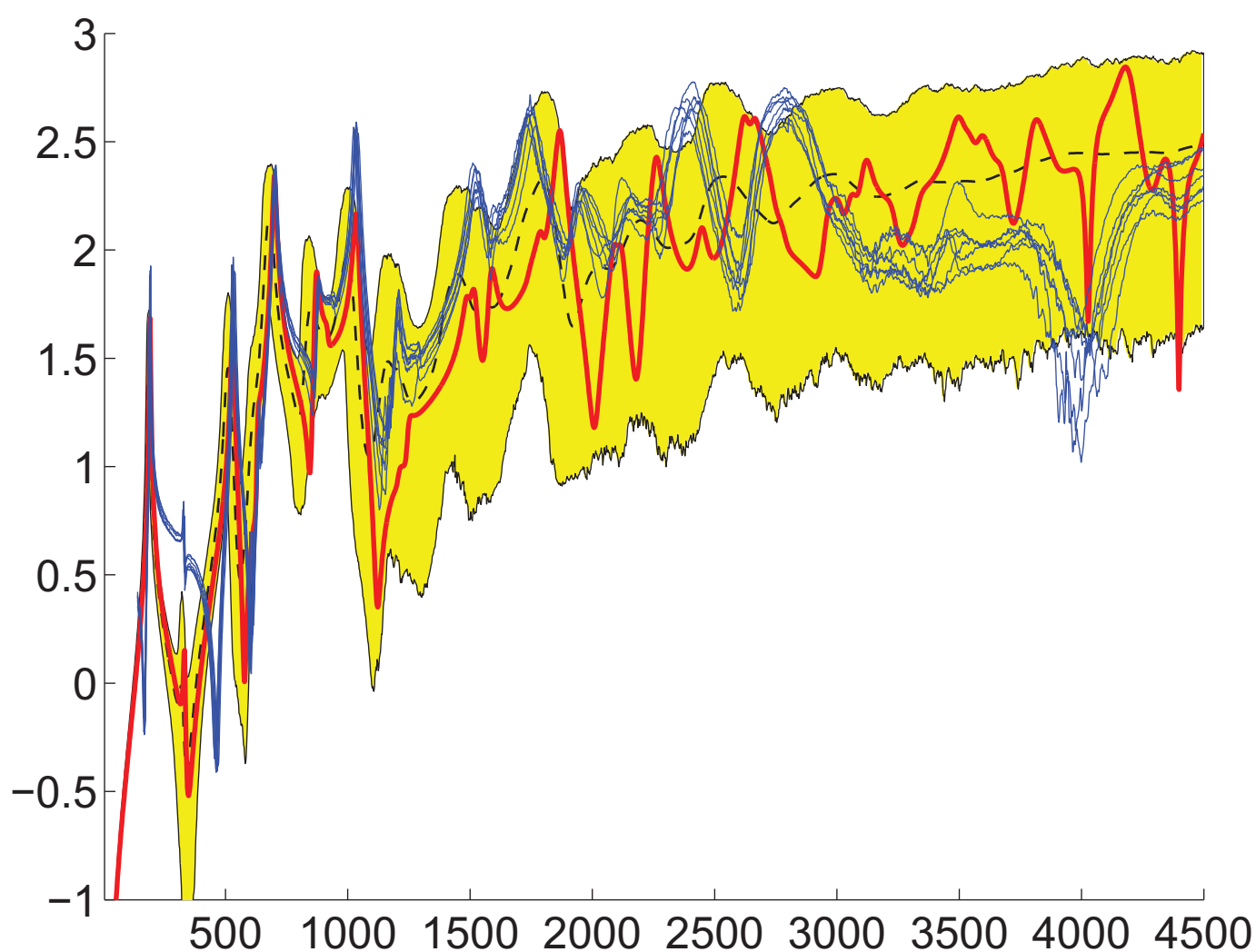

Figure 12 


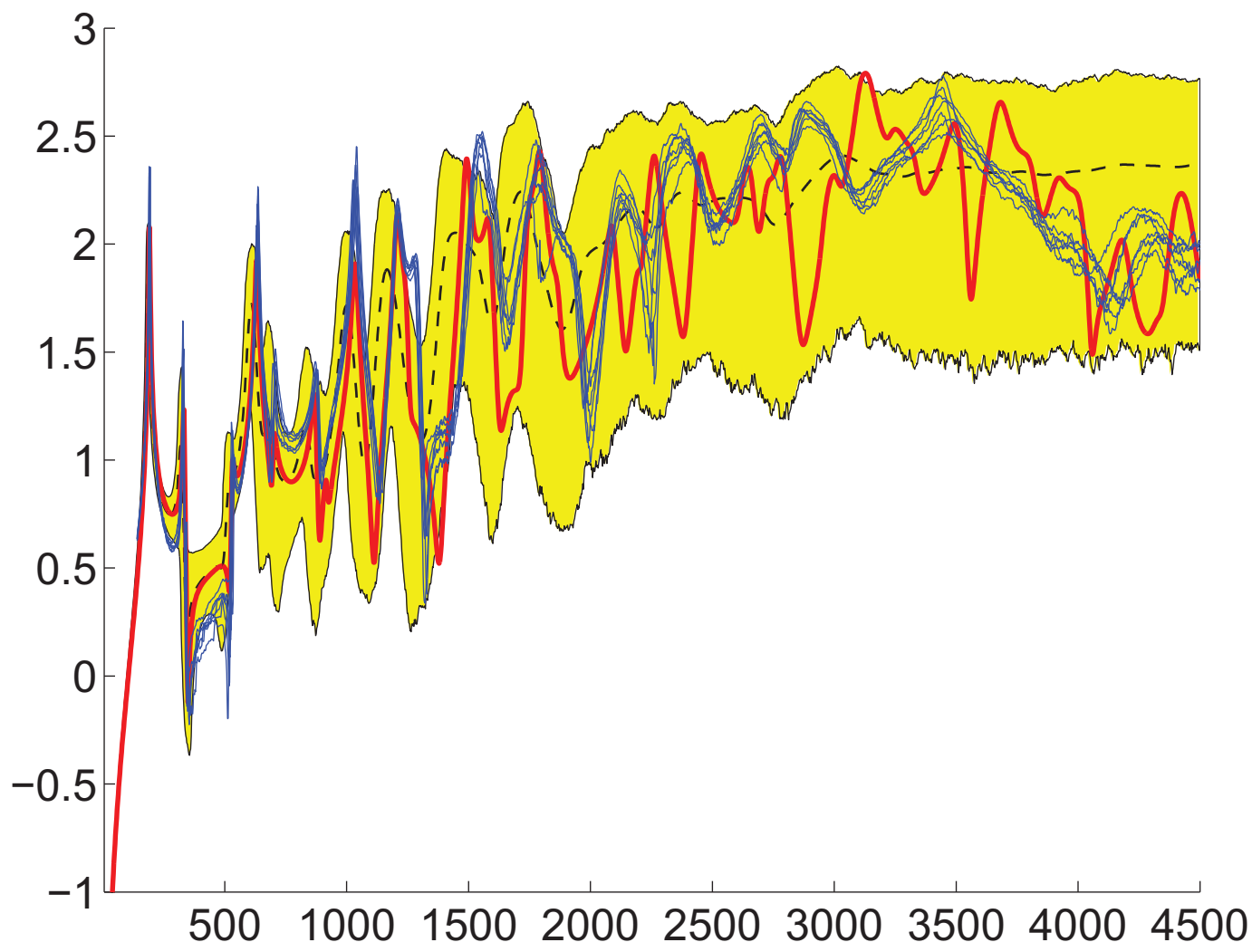

Figure 13 